ARTICLE

https://doi.org/10.1038/s41467-019-08555-w

\title{
The flavonoid 4,4'-dimethoxychalcone promotes autophagy-dependent longevity across species
}

Didac Carmona-Gutierrez et al."

Ageing constitutes the most important risk factor for all major chronic ailments, including malignant, cardiovascular and neurodegenerative diseases. However, behavioural and pharmacological interventions with feasible potential to promote health upon ageing remain rare. Here we report the identification of the flavonoid 4,4'-dimethoxychalcone (DMC) as a natural compound with anti-ageing properties. External DMC administration extends the lifespan of yeast, worms and flies, decelerates senescence of human cell cultures, and protects mice from prolonged myocardial ischaemia. Concomitantly, DMC induces autophagy, which is essential for its cytoprotective effects from yeast to mice. This pro-autophagic response induces a conserved systemic change in metabolism, operates independently of TORC1 signalling and depends on specific GATA transcription factors. Notably, we identify DMC in the plant Angelica keiskei koidzumi, to which longevity- and health-promoting effects are ascribed in Asian traditional medicine. In summary, we have identified and mechanistically characterised the conserved longevity-promoting effects of a natural anti-ageing drug. 
T he medical and socioeconomic advances experienced in developed countries over the last century have greatly extended life expectancy. However, health span has not increased at the same pace, resulting in the growing incidence and prevalence of age-related pathologies. Indeed, ageing remains the main risk factor for all major chronic maladies, including cardiovascular diseases, neurodegeneration and cancer $^{1}$. Since the majority of ageing people are polymorbid, even considerable advances against a single age-related disease may only marginally improve health span. Therefore, tackling age-onset diseases by targeting their commonality, the ageing process itself, appears the most expedient approach. To date, only a few efficient dietary or pharmacological anti-ageing interventions exist; these include calorie restriction (the permanent reduction of total caloric intake without malnutrition) and administration of pro-longevity drugs like spermidine, rapamycin, metformin, $\mathrm{NAD}^{+}$precursors, or resveratrol ${ }^{2,3}$. Further approaches that are able to regress (or at least delay) the onset of pathogenic age-related decline are urgently needed.

Interestingly, epidemiological studies suggest that the regular consumption of polyphenol-rich foods may decrease the risk of many chronic conditions ${ }^{4}$, and certain polyphenols-most prominently resveratrol-have been shown to extend life and/or health span in several model organisms ranging from yeast to mice $^{5}$. Polyphenols are phytochemicals widely dispersed throughout the plant kingdom with manifold functions in planta ranging from pollinator attraction to pathogen and UV protection. Among them, the flavonoids represent the largest polyphenol subgroup and many of them show anti-inflammatory, anti-carcinogenic, anti-neurodegenerative and general cytoprotective properties ${ }^{6,7}$. However, reports specifically addressing the long-term effects of chemically defined flavonoids on ageing remain rare.

Most if not all behavioural, nutritional, pharmacological, and genetic manipulations that are known to extend lifespan stimulate macroautophagy (hereafter referred to as autophagy). In fact, autophagy seems to be a causal effector of these protective characteristics. For instance, the longevity drugs resveratrol, rapamycin, and spermidine, all lose their efficacy when autophagy is suppressed ${ }^{2}$. Autophagy is an intracellular recycling process, in which damaged or superfluous macromolecules and organelles are sequestered in two-membraned vesicles (autophagosomes) and then targeted to lysosomes for bulk degradation ${ }^{8}$. This facilitates the supply of recycled components for biosynthesis and thus contributes to cytoplasmic renewal and consequent cellular rejuvenation. Conversely, impairment or dysregulation of autophagic function results in age-related pathologies ${ }^{9,10}$. Altogether, autophagy is largely associated with cytoprotection and overall health.

Here we report the identification of the flavonoid 4,4'-dimethoxychalcone (DMC) as a natural autophagy inducer with phylogenetically conserved anti-ageing properties. We found that administration of DMC promotes cytoprotection and autophagy across species and that autophagy induction is required for the beneficial effects of this compound. Autophagy activation by DMC depends on specific GATA transcription factors, but not on the TORC1 kinase, a major regulatory instance of autophagy. This suggests synergistic potential with other anti-ageing interventions that do rely on TORC1 signalling.

\section{Results}

4,4'-dimethoxychalcone (DMC) promotes longevity across species. In an effort to identify novel natural compounds with anti-ageing properties, we screened a library of 180 compounds representing different subclasses of flavonoids (Supplementary
Table 1) for their ability to counteract age-related cellular demise. For this purpose, we monitored cellular health during yeast chronological ageing-an established model for the ageing of human post-mitotic cells ${ }^{11-13}$-in the presence of each of these flavonoids at a concentration of $50 \mu \mathrm{M}$. Using a high-throughput approach (Fig. 1a, Supplementary Fig. 1a-e), we determined in parallel (i) cellular membrane integrity (survival) by means of propidium iodide (PI) staining (Fig. 1b, Supplementary Fig. 1d), (ii) the clonogenic potential (outgrowth) of aged cells (Fig. 1b, Supplementary Fig. 1e), and (iii) the production of reactive oxygen species (ROS) detectable as the ROS-driven conversion of dihydroethidium to fluorescent ethidium (Fig. 1c). In each of these three independent assays, DMC emerged as a top cytoprotective hit. Upon further determining the concentration dependency of DMC's rescuing effect, we established the optimal dose in yeast to be at $100 \mu \mathrm{M}$ (Supplementary Fig. 2a). DMC's potential to reduce chronological age-related cell death (as assessed by PI staining) was thereby comparable to that of several compounds previously reported as cytoprotective in ageing models. Precisely, DMC partly outperformed other polyphenols, including resveratrol and specific flavonoids (at $100 \mu \mathrm{M}$, as DMC), and yielded a similar protective capacity as rapamycin (at its optimal dosage of $40 \mathrm{nM}$ ) (Supplementary Fig. 2b). Confirming and extending our screening results, DMC treatment reduced the age-related increase in apoptotic and necrotic cell populations as determined by AnnexinV/PI co-staining, diminished the population of ROS-accumulating cells and promoted clonogenicity during ageing (Fig. 1d-f, Supplementary Fig. 2c, d).

We next examined whether DMC would act as an anti-ageing compound in two multicellular organisms, namely, the nematode Caenorhabditis elegans and the fruit fly Drosophila melanogaster. Remarkably, chronic DMC treatment $(41.6 \mu \mathrm{M}$ for worms, 200 $\mu \mathrm{M}$ for flies) prolonged the median lifespan of both model organisms by approximately 20\% (Fig. 1g, h, Supplementary Fig. 3a-k, Supplementary Table 2). Of note, DMC did neither affect food intake (Supplementary Fig. 4a) nor fecundity (Supplementary Fig. 4b) of flies, and its longevity-extending effects were independent of the food composition (Supplementary Fig. 4c-e). DMC $(50 \mu \mathrm{M})$ also largely prevented the senescencemediated decrease of clonogenic survival in highly confluent human cells (U2OS osteosarcoma, HeLa cervical carcinoma, and H4 neuroblastoma cells) (Fig. 1i, j, Supplementary Fig. 31). Altogether, these results suggest that DMC mediates phylogenetically conserved anti-ageing effects.

Autophagy is required for the beneficial effects of DMC. Most anti-ageing interventions depend on autophagy to exert their protective properties ${ }^{2}$, and several flavonoids have been reported to stimulate autophagy ${ }^{14}$. Thus, we tested whether DMC would activate autophagy. Indeed, in yeast cells subjected to chronological ageing, DMC increased the autophagy-dependent redistribution of a GFP-Atg8 fusion protein towards vacuoles and its subsequent degradation to yield free GFP (Fig. 2a-c, Supplementary Fig. $5 \mathrm{a}-\mathrm{c}$ ). Moreover, DMC treatment stimulated the activity of Pho8 $\Delta$ N60, a truncated form of alkaline phosphatase (ALP), the vacuolar delivery and activation of which depends on autophagy ${ }^{15,16}$ (Fig. 2d, Supplementary Fig. S5d, e). In worms, DMC feeding for $48 \mathrm{~h}$ resulted in increased formation of autophagosomes as indicated by microscopic analysis of mCherry-fused LGG-1, the worm homologue of Atg8 (Fig. 2e, f). Moreover, brains of aged flies fed with DMC for 30 days exhibited reduced abundance of $\operatorname{Ref}(2) \mathrm{P}$, the fly homologue of human sequestosome1 (SQSTM1/p62), suggesting increased autophagic flux, which generally leads to reduced abundance of $\operatorname{Ref}(2) \mathrm{P}^{17}$ (Fig. $2 \mathrm{~g}, \mathrm{~h}$ ). Similarly, DMC induced autophagic flux in several human cell 
lines (U2OS, colorectal carcinoma HCT116, hepatoma HepG2), as determined by immunochemical detection of LC3 lipidation (LC3-II) and microscopic detection of cytoplasmic GFP-LC3 dots in the absence or presence of chloroquine, which stalls lysosomal autophagosome degradation (Fig. 2i, j, Supplementary Fig. 6a-h). In addition, DMC caused a reduction of SQSTM1/p62 levels in HCT116 and HepG2 cells (Supplementary Fig. 6i-k). Finally, DMC administered intraperitoneally efficiently triggered autophagic flux in the heart and liver from wild type (WT) C57BL/6 mice, as shown by increased LC3 lipidation, even after leupeptin treatment, a lysosomal protease inhibitor (Fig. 3a, b,
Supplementary Fig. 6l, m). In conclusion, DMC induces autophagic flux in all model systems tested from yeast to mammals.

We next examined a possible causality between the induction of autophagy and the protective effects of DMC. DMC reduced the infarction area in mice subjected to prolonged myocardial ischemia (with no reperfusion), an autophagy-associated and ageing-relevant setting ${ }^{18-20}$, but only in wild type animals and not in mice with cardiac-specific autophagy deficiency (Fig. 3c, d, Supplementary Fig. 6n). Importantly, this dependence on autophagy also held true for the longevity phenotype. The knockout or knockdown of autophagy-related (ATG) genes, a

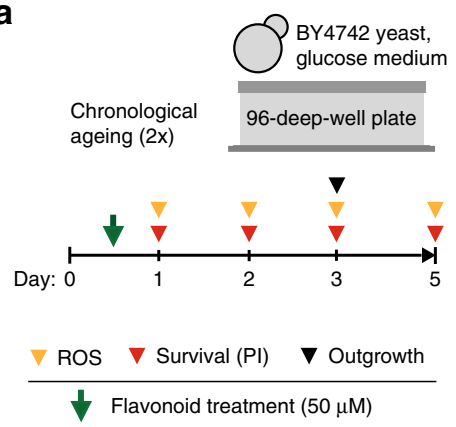

b

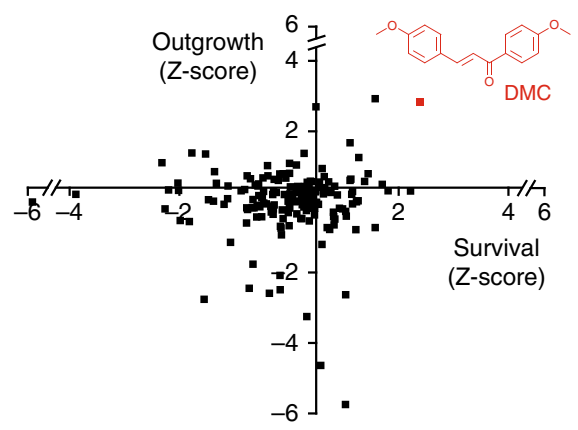

c

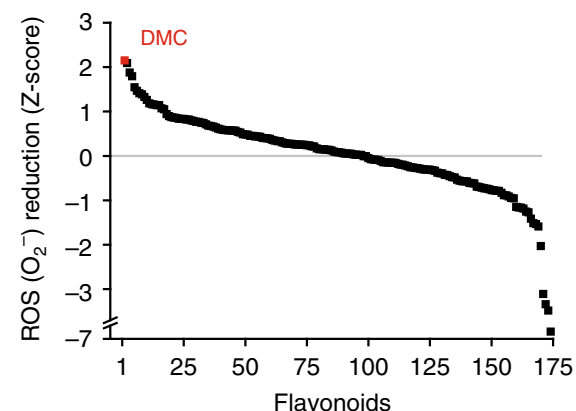

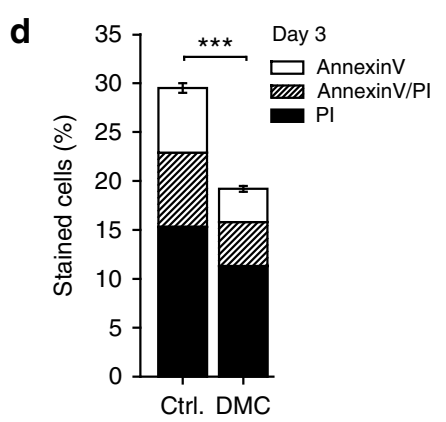

g

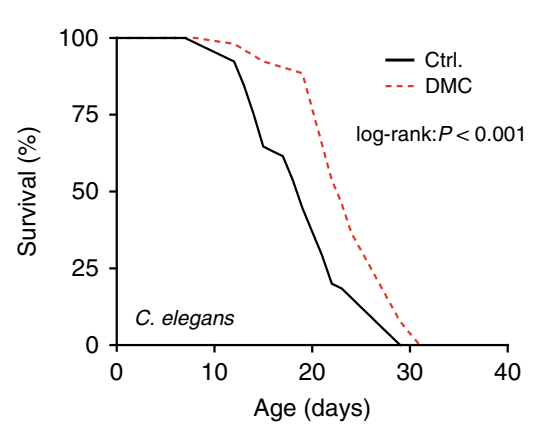

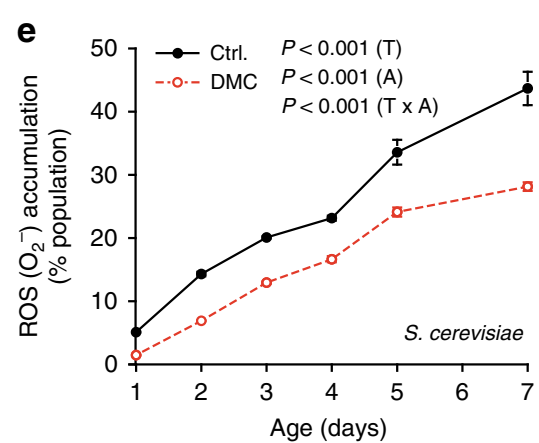

h

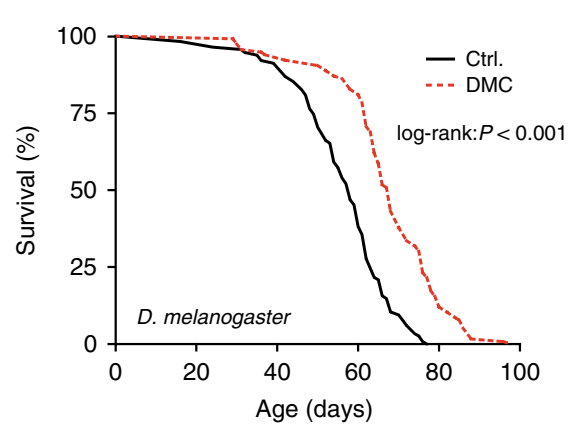

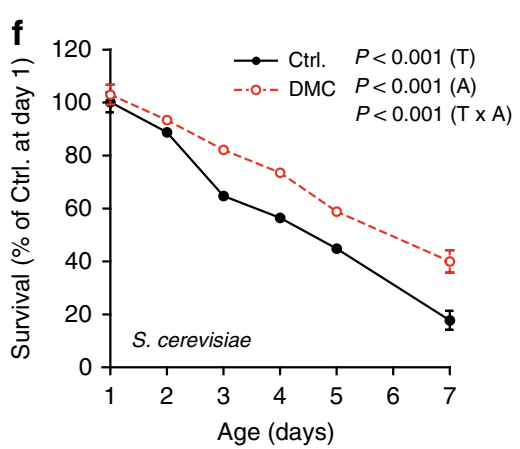

i Human U2OS j

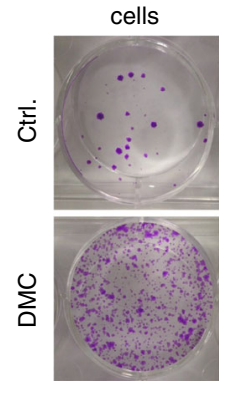

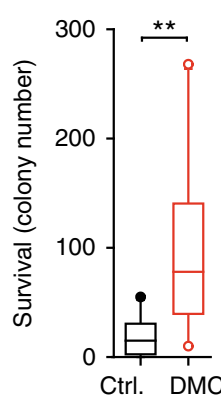

Fig. 1 4,4'-dimethoxychalcone promotes longevity in yeast, nematodes, flies and human cells. a Screening procedure for anti-ageing flavonoids in a yeast chronological ageing model. b, c Z-scores of AUCs obtained for each flavonoid during the yeast screen and for each assay performed: PI staining, outgrowth capacity (b), and DHE to ethidium conversion (c); data obtained in 1-2 independent runs with three replicates each. Each data point represents one flavonoid. The data point and the structure of 4, $4^{\prime}$-dimethoxychalcone (DMC) are shown in red. $\mathbf{d}$-f Phosphatidylserine externalisation and membrane dysintegrity (d), ROS production (e) and survival (f) of DMC-treated yeast cells (100 $\mu \mathrm{M})$ at indicated time points of chronological ageing using AnnexinV/PI costaining (d), ${ }^{\star \star \star} P<0.001, n=18$, DHE to ethidium conversion, $n=6$ (e) and clonogenicity, $n=8$ (f) independent biological replicates; $P$-values represent T, treatment; A, age; T $\times$ A, interaction. $\mathbf{g}$, h Survival of $C$. elegans ( $(\mathbf{g})$ or D. melanogaster (h) during ageing with supplementation of food with DMC ( $41.6 \mu \mathrm{M}$ for worms, $200 \mu \mathrm{M}$ for flies). For other ageing replicates, see Supplementary Figure 2 (yeast) and 3 (nematodes, flies). $\mathbf{i}$, $\mathbf{j}$ Replicative viability of DMC-treated $(50 \mu \mathrm{M})$ U2OS cells. Representative images (i) and quantification (j) are shown, ${ }^{\star \star} P=0.0069, n=11$ independent biological replicates. Data in (d) represent means \pm SEM; box plots in (j) represent IQR (line at median) and whiskers 10-90 percentile. Significance in (d, $\mathbf{j}$ ) was assessed by two-sided Student's $t$-test, in $(\mathbf{e}, \mathbf{f})$ by repeated measures two-way ANOVA. Source data for $(\mathbf{b}-\mathbf{f}, \mathbf{j})$ are provided as a Source Data file 
a

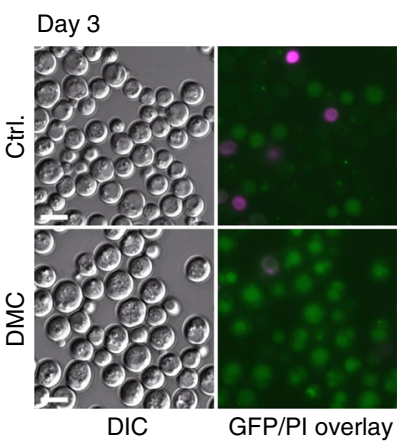

e

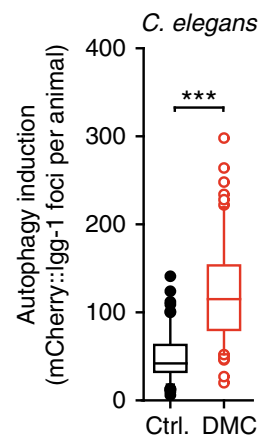

h

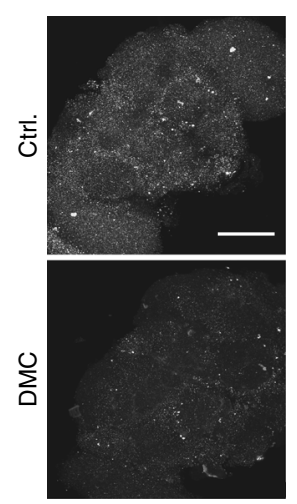

b

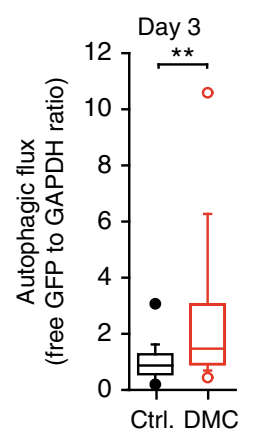

f

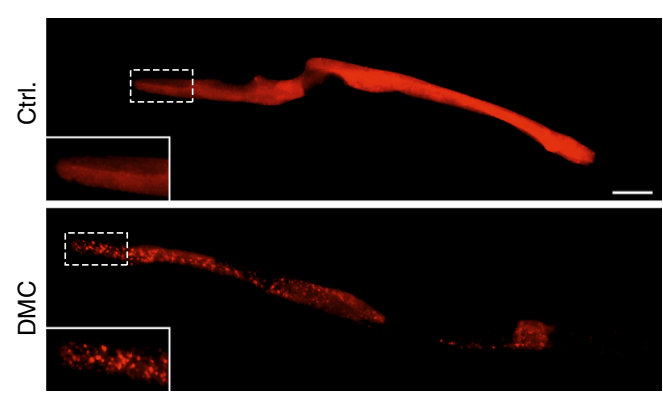

i

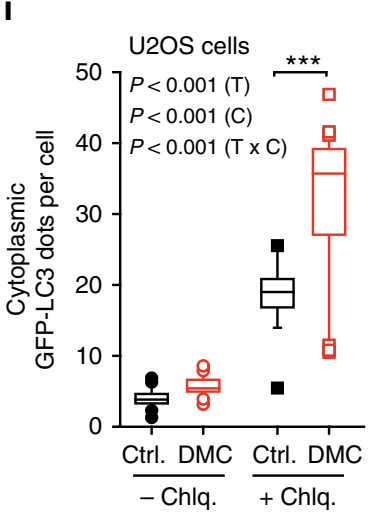

C

j

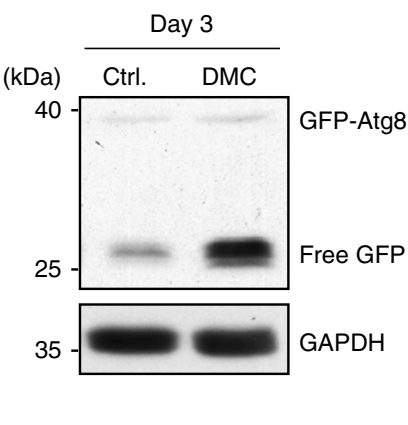

\section{g}

g D. melanogaster
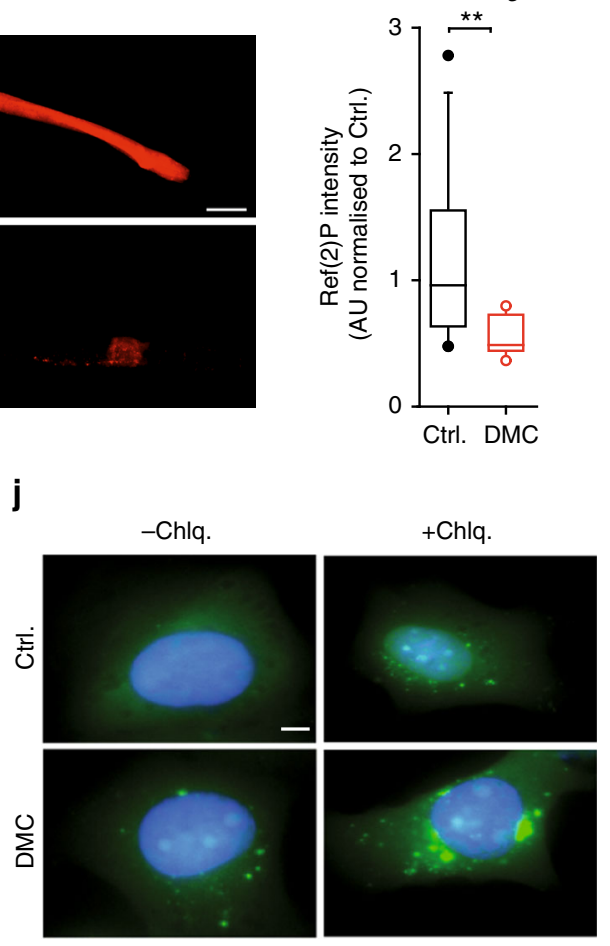

Fig. 2 4,4'-dimethoxychalcone induces autophagy across species. a-d Autophagic flux in DMC-treated (100 $\mu$ M) yeast indicated by the vacuolar accumulation of GFP-Atg8 (green); a representative micrograph is shown in (a), where propidium iodide (PI) counterstaining served to visualise dead cells (magenta). Corresponding quantification was performed by analysing the free GFP/GAPDH ratio (b). ${ }^{\star \star} P=0.0024, n=19$ (Ctrl.), 18 (DMC) independent biological replicates; c representative immunoblot. Autophagy measured via alkaline phosphatase (ALP) activity of Pho8 $\Delta$ N60 strains (d). ${ }^{\star} P=0.0020$, $n=11$ independent biological replicates. See Supplementary Figure $5 \mathrm{~d}$ for other time points. e, $\mathbf{f}$ Quantification (e) and representative pictures (f) of $\mathrm{mCherry}$-foci in the intestine cells of DMC-fed $(41.6 \mu \mathrm{M})$ young adult worms expressing Pnhx-2::mCherry::Igg-1. ${ }^{\star \star \star} P<0.0001, n=61(\mathrm{Ctrl}$.), 65 (DMC) animals. Scale bar: $50 \mu \mathrm{m} . \mathbf{g}, \mathbf{h}$ Immunofluorescence analysis $(\mathbf{g})$ and representative pictures (h) of ref. ${ }^{2}$ P-marked protein aggregates in fly brains after 30 days DMC feeding $(200 \mu M)$ ). ${ }^{\star} P=0.0026, n=12$ (Ctrl.), 11 (DMC) animals. Scale bar: $50 \mu \mathrm{m}$. Significance in (b, e, $\left.\mathbf{g}\right)$ was assessed by two-sided Student's $t$-tests, in (d) by ANOVA/Bonferroni. $\mathbf{i}$, $\mathbf{j}$ Videomicroscopic analysis (i) and representative pictures $(\mathbf{j})$ of DMC-treated $(50 \mu M) \cup 2 O S$ cells expressing GFP-LC3 with or without chloroquine, cell nuclei were stained with Hoechst 33342. Comparisons by two-way ANOVA (T, treatment; C, chloroquine; $T \times C$, interaction) followed by Bonferroni-corrected simple main effects, ${ }^{\star \star \star} p<0.0001, n=22$ (Ctrl.-Chlq), 21 (DMC-Chlq), 18 (Ctrl. + Chlq), 30 (DMC + Chlq) independent biological replicates. Scale bar: $10 \mu \mathrm{m}$. Box plots represent IQR (line at median) and whiskers 10-90 percentile. Source data for $(\mathbf{b}, \mathbf{d}, \mathbf{e}, \mathbf{g}, \mathbf{i})$ are provided as a Source Data file

which code for essential components of the autophagic machinery, abolished DMC-mediated lifespan extension in yeast, worms, and flies (Fig. 4, Supplementary Fig. 7a-h). Altogether, this indicates that autophagy induction is indeed necessary for the beneficial effects of DMC.

DMC targets the yeast GATA transcription factor Gln3. Next, we asked via which pathway(s) DMC might promote autophagy.
DMC did neither impair proteasome activity nor induce endoplasmic reticulum stress (Supplementary Fig. 8a, b), thus ruling out two possible xenobiotic-mediated, pro-autophagic routes ${ }^{21-23}$. We then decided to examine the anti-ageing effects of DMC in several yeast mutants deficient for genes/proteins known to be involved in autophagic signalling ${ }^{24}$ (Fig. 5a). The disruption of the GATA transcription factor GLN $3^{25}$ was the genetic intervention that most prominently abated DMC-mediated cytoprotection (Fig. 5a, b, Supplementary Fig. 8c, d). Of note, this phenotype 
a

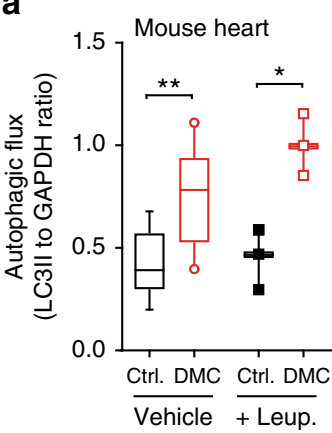

C

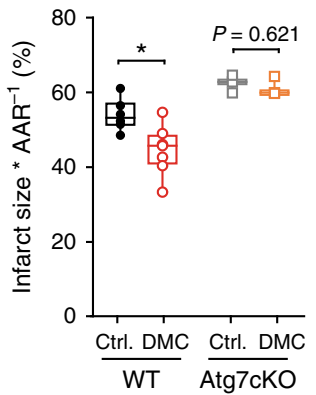

b

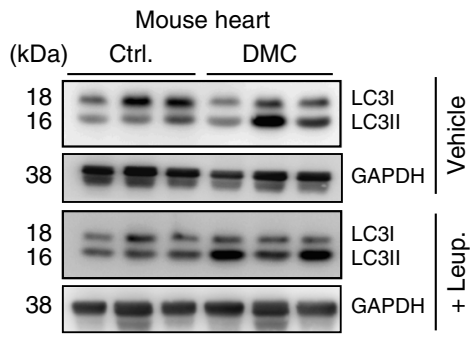

d

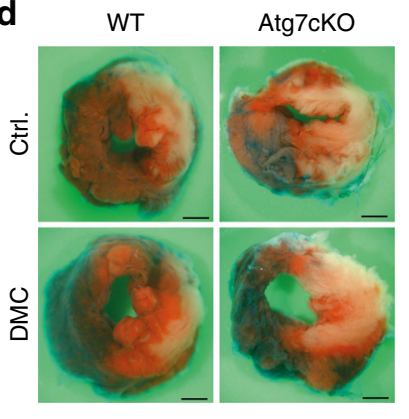

Fig. 3 4,4'-dimethoxychalcone promotes autophagy and cardioprotection in mice. a, b Autophagy induction in mouse heart tissue (a) determined by LC3 lipidation with leupeptin or vehicle injection after intraperitoneal injection of DMC (100 mg/kg) or DMSO (Ctrl.). $n=9$ (Ctrl. Vehicle), 10 (DMC Vehicle), 3 (Leup.) animals, ${ }^{\star} P=0.0105,{ }^{\star}{ }^{\star} P=0.0028$; b representative immunoblot. $\mathbf{c}$, $\mathbf{d}$ Infarction area per area at risk (AAR) (c) and representative images of left ventricular myocardial sections (scale bar: $1 \mathrm{~mm}$ ) (d) after DMC treatment as in (a-b) followed by $3 \mathrm{~h}$ prolonged ischaemia in wild type (WT) and cardiac-specific Atg7 knockout mice (Atg7cKO). ${ }^{\star} P=0.0134, n=5$ (Ctrl.), 6 (DMC), 3 (Atg7cKO) animals. Significance in $(\mathbf{a}, \mathbf{c})$ was assessed by two-sided Student's $t$-tests between Ctrl. and DMC. Box plots represent IQR (line at median) and whiskers 10-90 percentile. Source data for $(\mathbf{a}, \mathbf{c})$ are provided as a Source Data file

could be reversed by episomal Gln3 expression (Supplementary Fig. 8e, f), indicating that the observed effects were functionally linked to the deletion. DMC-mediated cytoprotection was also abrogated upon disruption of the Gln3 regulator protein phosphatase 2A (PP2A), either by deletion of its catalytic (PPH21/ $\mathrm{PPH} 22$ ) or one of its regulatory (TPD3) subunits (Fig. 5a). The three other yeast GATA transcription factors (Gat1, Dal80 and Gzf3) failed to affect DMC treatment (Supplementary Fig. 9). Consistently, DMC treatment could not induce autophagy in the absence of Gln3 (Fig. 5c, d). This suggests a crucial and specific role of this GATA transcription factor in the execution of DMC effects.

Gln3 is involved in the co-regulation of general amino acid control $^{26}$, and interference with amino acid metabolism has been linked to lifespan extension and autophagy induction across species $^{27-29}$. In accordance with an impact of DMC on Gln3 function, the metabolomic and proteomic profiles of DMCtreated yeast cells showed a pronounced influence on amino acid metabolism (Fig. 5e, Supplementary Fig. 10c, f, g). A similar metabolic repercussion was detected upon metabolomic analysis of heart and liver tissue of mice treated with DMC intraperitoneally (Supplementary Fig. 10a, b, d, e). Notably, the metabolic imprint of DMC-treated wild type yeast seems to be very similar to that of $g \ln 3$ deletion mutants both with respect to the amino acid profile $\mathrm{e}^{30}$ (Fig. 5e) and the metabolome in general (Fig. 5f). This suggests that the impact of DMC may suppress Gln3 activity. To test this, we monitored the expression of the $\beta$ galactosidase-encoding lac $Z$ gene placed under the control of the GIn3-inducible MEP2 promoter, which is specifically targeted by Gln $3^{31}$. In wild type yeast cells, DMC treatment reduced lacZ activity to levels close to those observed in the GLN3 knockout strain (Fig. 5g). This further supports the possibility that DMC mediates its effect by inhibiting Gln3 activity. We reasoned that in this case, the genetic disruption of GLN3 should per se also promote cytoprotection and autophagy. Indeed, GLN3 deletion mutants exhibited decreased age-related cell death and higher autophagy levels than wild-type cells (Fig. 5b-d, Supplementary Fig. 8c, d). Thus, Gln3 deletion and DMC treatment have similar, epistatic effects on yeast cells with respect to autophagy induction and cytoprotection, supporting the idea that both act on the same pathway. Altogether, our data argue for an anti-autophagic role of a

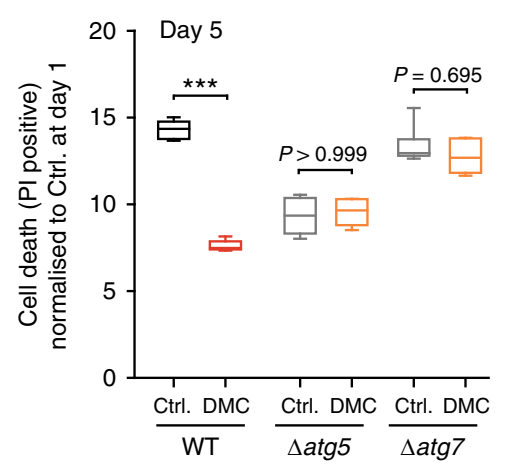

b

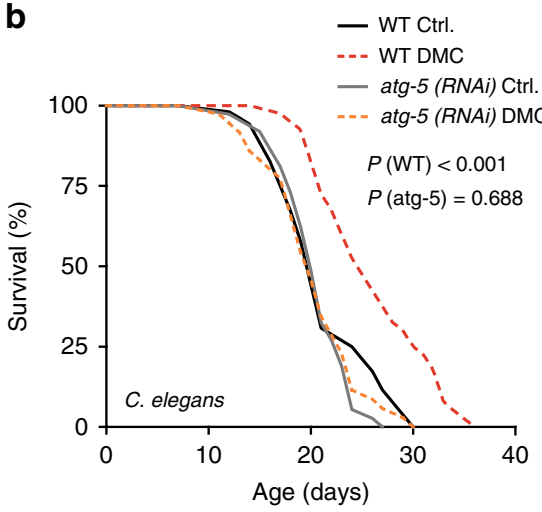

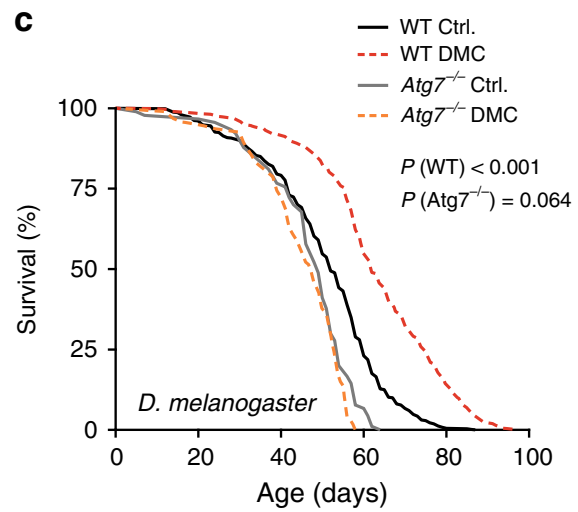

Fig. 4 Autophagy induction is essential for 4,4'-dimethoxychalcone-mediated protection. a Survival of DMC-treated (100 $\mu$ M) yeast wildtype (WT), ATG5-deficient, and ATG7-deficient mutant strains at day 5 of chronological ageing measured by PI staining. Data are normalised to the WT Ctrl. at day 1. Comparisons by two-way ANOVA with treatment and strain as independent variables, followed by Bonferroni-corrected simple main effects. ${ }^{\star \star \star} P<0.001, n=6$ independent biological replicates. Box plots represent IQR (line at median) and whiskers 10-90 percentile. Refer to Supplementary Figure 7 for other time points and other replicate experiments. Source data for (a) are provided as a Source Data file. b, c Survival of DMC-fed $(41.6 \mu \mathrm{M})$ control and autophagy-deficient Atg5 RNAi nematodes (b) or female wildtype (WT) and Atg7-deficient (Atg7-/-) mutant flies (c) during ageing. Refer to Supplementary Figure 7 for other replicate experiments. P-values represent pairwise comparisons (Ctrl. vs. DMC) by logrank analysis 
a

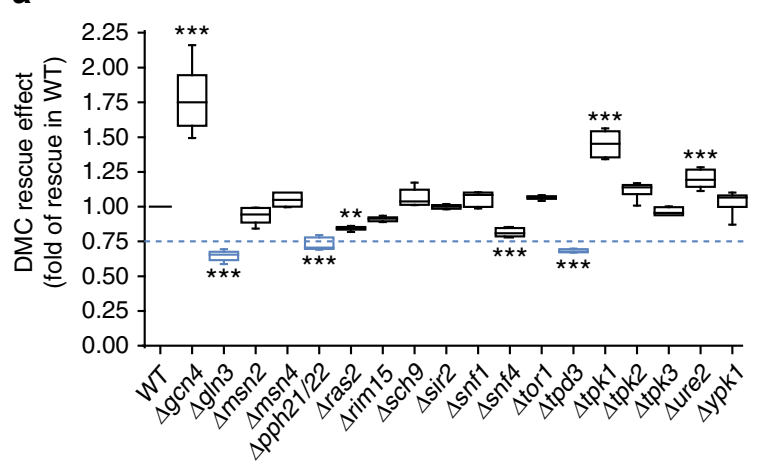

b

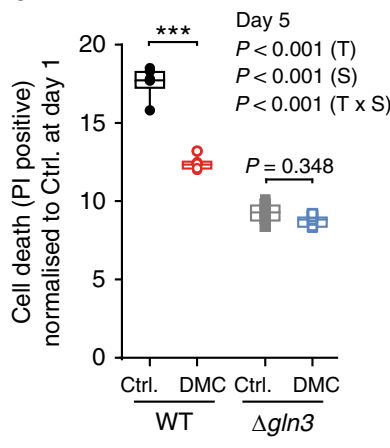

f

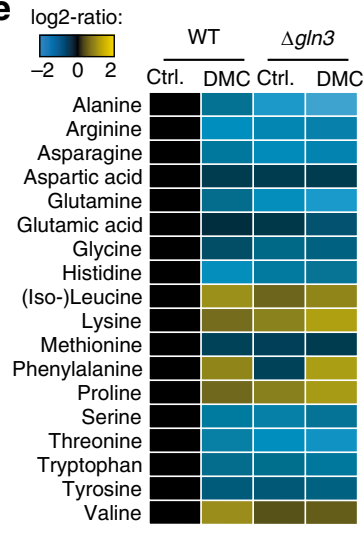

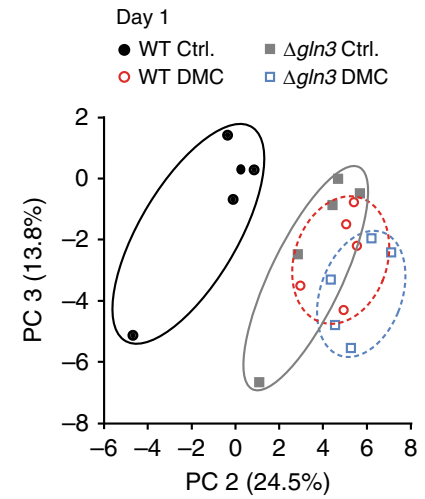

C

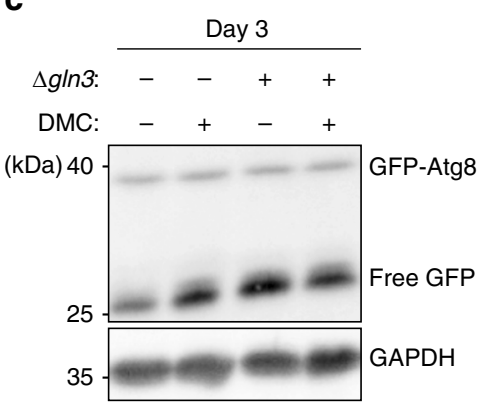

d

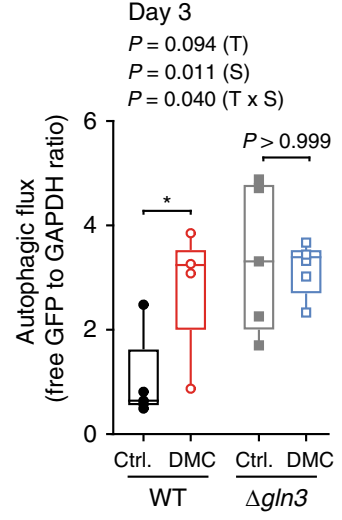

Fig. $5 \mathrm{Gln} 3$ is a functional target of 4,4'-dimethoxychalcone. a DMC-rescuing effect determined as the AUC of PI-positive cells (day 1-5) upon DMC treatment $(100 \mu \mathrm{M})$ of yeast deletion mutants involved in autophagic signalling, normalised to the rescuing effect in wildtype (WT) cells. Hits below the threshold (0.75) are depicted in blue. Comparisons by ANOVA/Bonferroni. ${ }^{\star \star} P<0.01,{ }^{\star \star \star} P<0.001, n=6$ independent biological replicates. $\mathbf{b}$ Cell death at day 3 of chronological ageing of DMC-treated $(100 \mu \mathrm{M})$ yeast wildtype (WT) and GLN3-deficient mutant strains determined by PI staining normalised to Ctrl. at day $1(\mathbf{b}),{ }^{\star \star \star} P<0.001, n=6$ independent biological replicates. (c, d) Autophagy induction of DMC-treated (100 $\left.\mu M\right)$ yeast wildtype (WT) and GLN3-deficient mutant strains indicated by the free GFP to GAPDH ratio normalised to WT Ctrl. (d), ${ }^{\star} P=0.0242, n=5$ independent biological replicates; representative immunoblot in (c). e, $\mathbf{f}$ Changes in intracellular amino acid concentrations (e) and principal component analysis of yeast metabolites (f) $24 \mathrm{~h}$

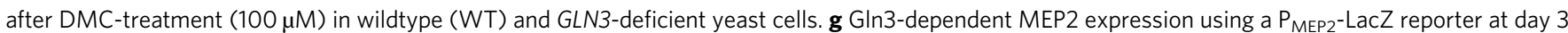
after DMC-treatment $(100 \mu \mathrm{M})$ in wildtype (WT) and GLN3-deficient yeast cells. ${ }^{\star \star} P=0.0056, n=12(\mathrm{WT}) n=9$ ( $\Delta$ g/n3) independent biological replicates; Comparisons in ( $\mathbf{b}, \mathbf{d}, \mathbf{g}$ ) by two-way ANOVA ( $\mathrm{T}$, treatment; $\mathrm{S}$, strain; $\mathrm{T} \times \mathrm{S}$, interaction) followed by Bonferroni-corrected simple main effects. Box plots represent IQR (line at median) and whiskers 10-90 percentile. Source data for (a, b, d-g) are provided as a Source Data file

Gln3 during chronological ageing that can be suppressed by DMC.

DMC acts independently of TORC1. We next asked how DMC might target Gln3 activity. The classical model of Gln3 cytoplasmic-nuclear translocation and activation involves TORC1-dependent negative regulation ${ }^{32}$. Thus, TORC1 inhibition, which is consistently connected to longevity ${ }^{33}$, actually stimulates Gln3 activity and does not suppress it as DMC. Accordingly, treatment with rapamycin, an inhibitor of the TORC1 kinase complex with well-established anti-ageing effects, promoted activation of the Gln3-inducible MEP2 promoter, and this effect was completely lost upon GLN3 deletion as previously described $^{31}$ (Supplementary Fig. 11a). Intriguingly, however, rapamycin continued to mediate both cytoprotection and autophagy induction under these GLN3-defective conditions. Deletion of Gln3 did neither preclude the reduction of agedependent cell death (as determined by PI staining) nor the stimulation of autophagic flux (as determined by GFP liberation) induced by rapamycin (Fig. 6a-c, Supplementary Fig. 11b, c). These results indicate that DMC and rapamycin mediate cytoprotection via independent pathways. Consequently, the combinatorial treatment with both agents, which consistently reduced Gln3 activity compared to rapamycin supplementation alone (Supplementary Fig. 11d), resulted in additive cytoprotective effects (Supplementary Fig. 11e). Thus, DMC seems to operate independently from TORC1. Indeed, neither deletion of the main TORC1 component TOR1 nor YPK1, a downstream target kinase of TORC2 involved in the positive regulation of autophagy during amino acid starvation ${ }^{29}$, influenced DMC effects (Fig. 5a). In line, as opposed to rapamycin, DMC did not influence ribosomal protein S6 (Rps6) phosphorylation, a downstream marker of TORC1 activity (Fig. 6d; Supplementary Fig. 11f). These data suggest that DMC regulates Gln3 activity in a manner that differs from the classical TORC1-dependent route.

We further unveiled a functional interaction of DMC with the Gln3 regulator PP2A. Its genetic inactivation by deletion of both its functionally redundant catalytic subunits ( $\mathrm{PPH} 21, \mathrm{PPH} 22)$ receded DMC effects (Fig. 5a, Supplementary Fig. 11g). These catalytic subunits can form distinct complexes with multiple regulatory/specificity subunits relevant for $\mathrm{Gln} 3$ regulation. One of these complexes is formed with the scaffold module Tpd3, the deletion of which we found to revert DMC-mediated effects (Fig. 5a, Supplementary Figure 11g), similar to PPH21/22 disruption. Notably, this specific complex-as opposed to the 


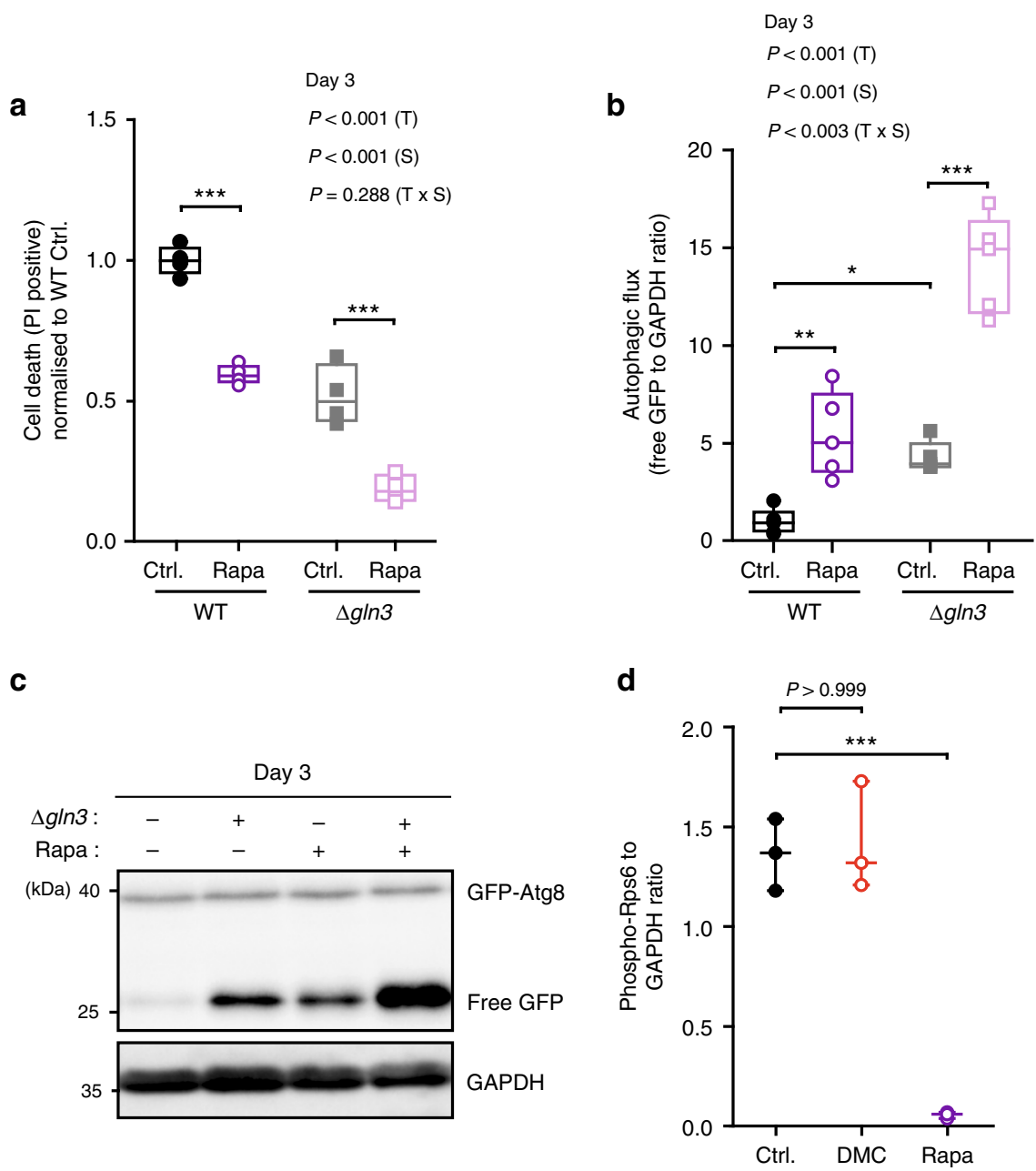

Fig. 6 Rapamycin and 4,4'-dimethoxychalcone follow independent routes of cytoprotection. a-c Cell death assessed by PI staining and flow cytometry normalised to WT Ctrl. (a) and autophagy induction indicated by the free GFP to GAPDH ratio normalised to WT Ctrl. b, c of S. cerevisiae wildtype and GLN3-deficient mutant strains after treatment with $40 \mathrm{nM}$ rapamycin (Rapa) at day 3 of chronological ageing. Comparisons in (a, b) by two-way ANOVA ( $T$, treatment; S, strain; TXS, interaction) followed by Bonferroni-corrected simple main effects. ${ }^{\star} P<0.0483,{ }^{\star \star} P<0.0055,{ }^{\star \star \star} P<0.001, n=4(\mathbf{a}), 5$ (b) independent biological replicates. (d) Rps6 S232/S233 phosphorylation of S. cerevisiae wild-type cells after $6 \mathrm{~h}$ of DMC (100 $\mu$ M) or $40 \mathrm{nM}$ rapamycin (Rapa) treatment as determined by immunoblotting. Comparisons by ANOVA/Bonferroni. ${ }^{\star \star \star} P<0.001, n=3$ independent biological replicates. Box plots represent IQR (line at median) and whiskers 10-90 percentile. Source data for $(\mathbf{a}, \mathbf{b}, \mathbf{d})$ are provided as a Source Data file

one with Tap42-is rapamycin-insensitive ${ }^{34,35}$, supporting the idea that TORC1 is not involved in DMC effects. In aggregate, our data suggest that DMC functionally targets Gln3 in a TORC1-independent manner.

GATA transcription factors are conserved DMC effectors. Finally, we tested whether the described dependence of DMC on yeast Gln3 is phylogenetically conserved. Indeed, silencing of the C. elegans GATA transcription factor elt-1, a Gln3 homolog, precluded both DMC-mediated lifespan extension and autophagy induction in worms (Fig. 7a-c, Supplementary Fig. 12a, b). Similarly, elt-1 knockout animals treated with DMC did not show improved survival during ageing (Supplementary Fig. 12c-e). Intriguingly, in human U2OS cells, siRNA-mediated silencing of GATA2 (and to a lower extent also that of GATA3, but not that of other Gln3 homologues), precluded autophagy induction by DMC (Fig. 7d, e, Supplementary Fig. 13). In contrast, rapamycininduced autophagy remained unaltered upon GATA2 silencing (Supplementary Fig. 14a), again supporting the concept that DMC and rapamycin ignite independent pathways across species. In fact, DMC-as opposed to rapamycin-did not inhibit
TORC1 signalling as determined by sustained S6K1 phosphorylation levels (Supplementary Fig. 14b). In aggregate, these results indicate a conserved role for specific Gln3-like GATA transcription factors in DMC-mediated autophagy and lifespan extension.

\section{Discussion}

While the beneficial effects of certain behavioral and dietary strategies (especially calorie restriction) are uncontestable ${ }^{2}$, most individuals have difficulties to strictly and permanently adhere to them. This has encouraged the search for potential pharmacological alternatives. The present work identifies the flavonoid $4,4^{\prime}$ dimethoxychalcone (DMC) as an anti-ageing compound with cardioprotective effects in mice and the potential to promote longevity across species. This echoes prior studies reporting that chalcones possess a wide-though poorly defined-range of biological activities relevant to human health ${ }^{6}$. So far, DMC has been only reported to mediate a mild anti-malarial $\left(\mathrm{IC}_{50}\right.$ $21.7 \mu \mathrm{M})^{36}$ activity and to inhibit the proliferation of human $\mathrm{K} 562$ leukaemia cell lines $\left(\mathrm{IC}_{50} 15 \mu \mathrm{M}\right)^{37}$. To our knowledge, there is no natural source of DMC known to date. Intriguingly, 

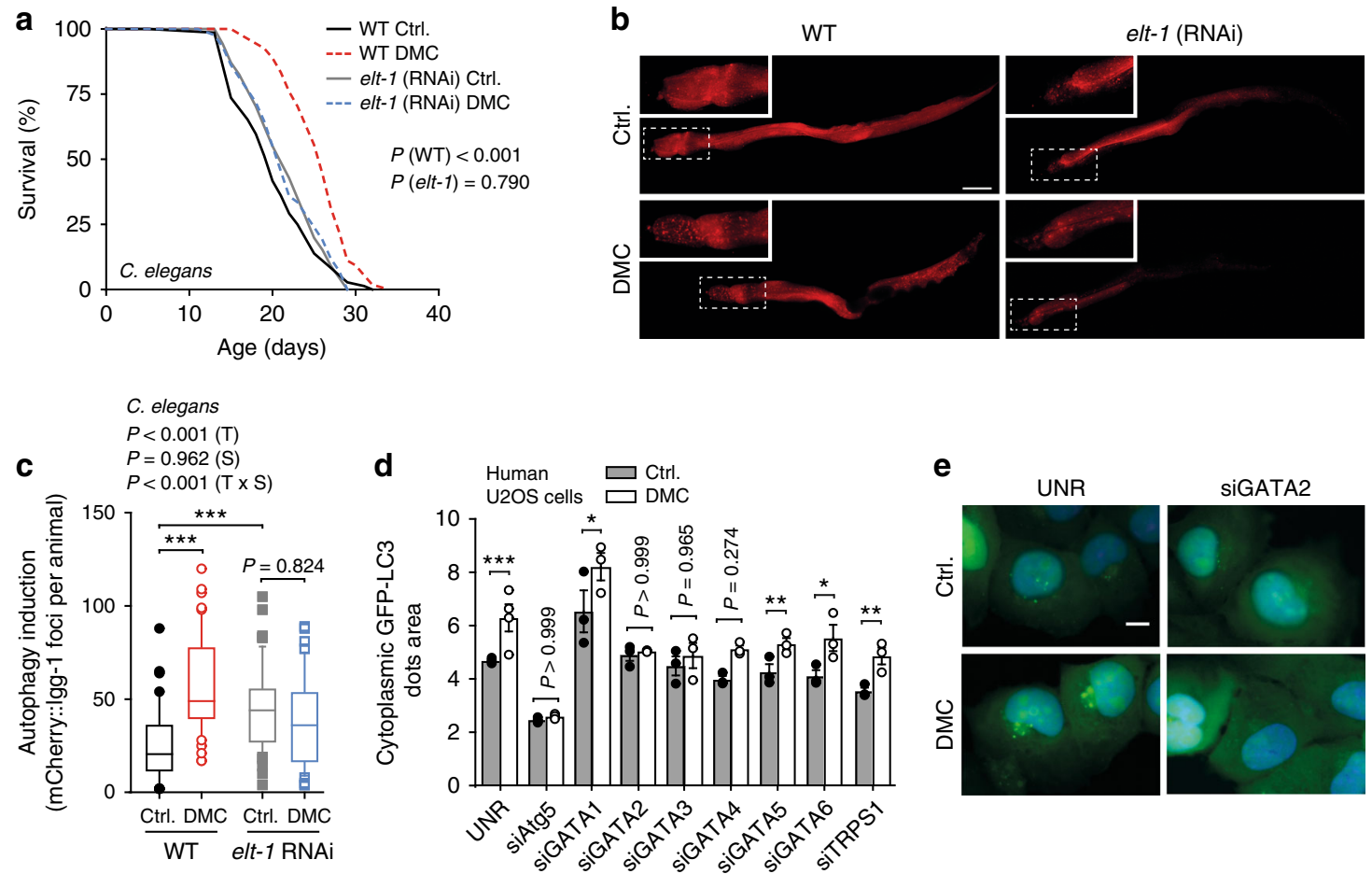

Fig. 7 GATA transcription factors are phylogenetically conserved effectors of 4,4'-dimethoxychalcone. a Survival of DMC-fed (41.6 $\mu$ M) control and GATA transcription factor-deficient elt-1 RNAi nematodes. P-values represent pairwise comparisons by log-rank analysis. Refer to Supplementary Figure 12 for other replicate experiments. b, c Representative fluorescence pictures (b) and quantification (c) of mCherry-positive foci in the intestine cells of DMC-fed $(41.6 \mu \mathrm{M})$ or untreated control or GATA-factor-deficient elt-1 (RNAi) nematodes expressing $\mathrm{P}_{\text {nhx-2: }}$ :mCherry::Igg-1 reflective of autophagosome generation. Comparisons by two-way ANOVA (T, treatment; $S$, strain; TxS, interaction) followed by Bonferroni-corrected simple main effects. ${ }^{\star \star \star} P<0.001, n=44$ (WT Ctrl.), 53 (WT DMC), 57 (elt-1 Ctrl.), 51 (elt-1 DMC) animals. Scale bar: $50 \mu \mathrm{m}$. Box plots represent IQR (line at median) and whiskers 10-90 percentile. d, e Autophagy induction in DMC-treated ( $50 \mu \mathrm{M})$ human U2OS cells with siRNA against an unrelated sequence (UNR) or GATA transcription factors as determined via videomicroscopy of cells expressing GFP-LC3 (d). Cell nuclei were stained with Hoechst 33342. Data shown as means of three different siRNA constructs \pm SEM. Comparisons by ANOVA with Tukey correction. ${ }^{\star} P<0.05,{ }^{\star \star} P<0.001,{ }^{\star \star \star} P<0.001, n=4$ (UNR), 3 (others) independent biological replicates. Representative images are shown in (e). Scale bar: $10 \mu \mathrm{m}$. Source data for (c, $\mathbf{d})$ are provided as a Source Data file

we could detect DMC in the stipes and leaves (but not in the roots) of the chalcone-rich plant Angelica keiskei koidzumi (commonly known under the Japanese name of Ashitaba), to which longevity- and health-promoting effects are attributed in Asian folk medicine (Supplementary Fig. 15a, b). This fuels the expectation that DMC may be therapeutically applicable in humans. Incidentally, DMC seems to be well tolerated in mice with no apparent side effects or toxicity, at least up to a dose level of $2000 \mathrm{mg} / \mathrm{kg}$ per os during an observation time of 14 days $^{38}$. Moreover, we could detect DMC in the blood plasma of middleaged mice that were fed with chow containing $0.25 \%$ DMC for 7 days, suggesting that orally administered DMC becomes bioavailable in mammals (Supplementary Fig. 15c).

Our data further demonstrate that the protective effects of DMC are mediated by autophagy induction. Intriguingly, the anti-ageing capacity of most interventions-independently of their upstream targets-seems to converge in autophagy induction. Still, many flavonoids have antioxidant potential that might mediate some degree of acute cytoprotection separately from autophagy. For instance, DMC protected mice against hepatotoxicity induced by acute ethanol intoxication, as measured by decreased serum alanine aminotransferase (ALT) activity, in both wild type and whole body ATG4B knockout animals (Supplementary Fig. 6o). Along similar lines, DMC slightly reduced cell death during the early phase of chronological ageing in different yeast strains, were they autophagy-competent or not (Supplementary Fig. 7h). However, at later time points, the anti-ageing effects of DMC were largely lost in autophagy-deficient yeast strains. In fact, the antioxidant capacity does not solely determine the long-term effects of cytoprotective flavonoids, as indicated by the fact that several flavonoids with powerful antioxidant activity ${ }^{39}$ failed to mediate anti-ageing effects in our initial screen (Supplementary Fig. 2e). Accordingly, other studies have shed doubts on the exclusive relationship between the healthpromoting and antioxidant properties of flavonoids ${ }^{40}$.

DMC promotes autophagy via a pathway that involves specific GATA transcription factors. In yeast, our results suggest that during chronological ageing, the GATA transcription factor Gln3 limits survival by exerting an anti-autophagic role, and that this can be reverted by DMC. While a previous genetic highthroughput study, in which $\Delta g \ln 3$ cells were found to be longlived $^{41}$, supports this notion, the role of $\mathrm{Gln} 3$ in autophagy seems to be more controversial. In discrepancy with our data, Gln3 has previously been shown to promote ATG14 expression upon nitrogen starvation in the logarithmic phase, raising the presumption that it might have a general pro-autophagic role ${ }^{42}$. However, this work did not measure actual autophagic flux in gln3-deficient strains, and ATG protein expression levels alone are not an accurate means to monitor autophagy ${ }^{15,43}$. In addition, a more recent study has challenged these observations by not finding any changes in ATG14 transcription or autophagy induction in $g \ln 3$ deletion mutants subjected to nitrogen starvation, although other ATGs seemed to be upregulated ${ }^{44}$. More importantly, this latter report also showed that under basal autophagic conditions in the logarithmic phase, the deletion of GLN3 (but not GAT1) actually promoted accumulation of ATG8 
and ATG29 mRNAs (ATG14 remained unaffected). ${ }^{44}$ The authors of that study concluded that Gln3 must have a direct or indirect role in the repression of some ATG genes when autophagy functions at basal/physiological levels ${ }^{44}$. Accordingly, we observed increased expression levels of different ATG proteins in ageing $\Delta g \ln 3$ cells (Supplementary Fig. 16). While under acute autophagy induction, e.g. upon nitrogen starvation, Gln3 might positively contribute to the expression of distinct $\mathrm{ATGs}^{44}$, our data and the recent literature ${ }^{41,44}$ are consistent with a role for Gln3 in restraining cytoprotective autophagy operating at a basal activity, at least during physiological chronological ageing conditions. We thus surmise that, by interfering with the autophagyrepressive activity of Gln3, DMC can increase autophagic flux thus conferring cytoprotection upon ageing.

Both Gln 3 activation ${ }^{32}$ and lifespan extension ${ }^{33}$ are associated to inhibition of TORC1. However, we show here that these two outcomes are correlative, i.e. that $\mathrm{Gln} 3$ activation as it occurs during TORC1 inhibition is not causal for cytoprotection. Similarly, our results support the notion that Gln3 is not required for rapamycin-mediated autophagy induction, as GLN3 deletion and rapamycin treatment induce autophagy in an additive manner. These data suggest, on the one hand, that DMC regulates Gln3 activity TORC1-independently. Indeed, recent evidence indicates that such non-canonical mechanisms of Gln3 regulation may exist ${ }^{45-47}$. On the other hand, it establishes synergistic potential with other anti-ageing interventions that act on the TORC1 inhibitory pathway. As a proof of principle, we show that DMC and rapamycin (a specific TORC1 inhibitor) exert additive cytoprotective effects.

Several GATA transcription factors have been connected to lifespan control ${ }^{48}$ in higher eukaryotes, and we can show that-as in yeast-DMC effects depend on specific GATA transcription factors in nematodes and human cell cultures. It should be noted that in worms, silencing of the GATA transcription factor elt-1 did not per se promote organismal lifespan extension (as opposed to yeast Gln3), although it did increase autophagy in intestinal cells. This might be due to tissue-restricted effects of elt-1 RNAi or due to a higher complexity of longevity regulation in higher eukaryotes through interactions among several GATA family members ${ }^{49}$. In mammalian cells, the role of GATA transcription factors in autophagy is only starting to be elucidated with differing outcomes depending on the specific factor. Only two reports have addressed this issue ${ }^{50,51}$. On the one hand, GATA1 has been shown to promote autophagosome formation and transcription of genes encoding ATG components in human erythroblasts, although the effects on different ATGs were rather heterogeneous ${ }^{50}$. On the other hand, GATA4 seems to inhibit doxorubicin-mediated autophagy in rat cardiomyocytes ${ }^{51}$. Our data identifies GATA2 as a further family member with antiautophagic capacity, at least under the tested conditions.

In the last century, life expectancy has increased globally, resulting in a demographic transformation characterised by an increased ageing population. This has greatly boosted the incidence and prevalence of late onset afflictions, which represents a pressing socioeconomic concern. Thus, it is critical to devise feasible anti-ageing interventions. The present work establishes the potential of DMC as a pharmacological instrument against ageing and age-related diseases. Future studies must explore whether DMC and/or its chemically defined derivatives can be advantageously used in humans as well.

\section{Methods}

Reagents. The following reagents were purchased from the indicated suppliers: FITC-labeled Annexin V (Roche Applied Science [11828681001]), diyhdroethidium (DHE, Sigma-Aldrich, [D7008]), propidium iodide (PI, SigmaAldrich [P4170]), 4,4'-dimethoxychalcone (DMC; ABCR, [AB179040];
Extrasynthese, [1295]; Sigma-Aldrich, [S617237]), rapamycin (LC laboratories, [R5000]), resveratrol (Sigma-Aldrich [R5010]). For the initial yeast screen, all flavonoids (including 4,4'-dimethoxychalcone) were purchased from Extrasynthese; for a complete list including the name, subclass and article number of each flavonoid please refer to Supplementary Table 1. Flavonoid stock solutions were always freshly prepared in DMSO prior to treatment. Acetonitrile (Chromasolv), formic acid (puriss), ethylacetate (Chromasolv) were purchased from Sigma Aldrich (St Lois, USA). Water was purified by MilliQ system $(<18.2 \mathrm{M} \Omega \mathrm{cm}$, Merck, Darmstadt, Germany).

Yeast strains and plasmids. Experiments were carried out in BY4742 (MATa his $3 \Delta 1$ leu $2 \Delta 0$ lys $2 \Delta 0$ ura $3 \Delta 0$ ) and respective null mutants, obtained from Euroscarf or self-generated (Supplementary Table 3) as described below. To monitor subcellular localisation of endogenous Atg8, a previously reported strain expressing an EGFP-Atg8 fusion protein was used ${ }^{52}$. Atg protein expression was determined using chromosomal 6(HA)-tagged strains, generated using pYM17 as a template ${ }^{53}$ All strains were grown on SC medium containing $0.17 \%$ yeast nitrogen base (BD Diagnostics, Schwechat, Austria), $0.5 \%\left(\mathrm{NH}_{4}\right)_{2} \mathrm{SO}_{4}, 30 \mathrm{mg} / \mathrm{l}$ of all amino acids (except $80 \mathrm{mg} / \mathrm{l}$ histidine and $200 \mathrm{mg} / \mathrm{l}$ leucine), $30 \mathrm{mg} / \mathrm{l}$ adenine and $320 \mathrm{mg} / \mathrm{l}$ uracil, with $2 \%$ glucose (SCD). For complementation experiments with Gln3, i.e. for episomal Gln3 expression, cells were grown on SCD as described below and upon flavonoid treatment galactose was added to a final concentration of $0.1 \%$ to induce expression. Successful expression was verified via western blot; note that the double band obtained (Supplementary Fig. 8f) is characteristic for Gln $3^{54}$.

Yeast chronological ageing experiments. Chronological ageing experiments were carried out in 96-deepwell plates (Bel-Art Products, USA), sealed with gaspermeable adhesive membranes (Excel Scientific) and lids. Therefore, $500 \mu \mathrm{l}$ of fresh media were inoculated with overnight cultures from cellular material of a 4day-incubated YEPD plate to an $\mathrm{OD}_{600}$ of $0.1\left(\sim 1.10^{6}\right.$ cells $\left.\mathrm{ml}^{-1}\right)$. Thereby, the wells at the outer rims of the deepwell plates were not inoculated, since they are prone to dry out in the course of ageing; instead they were loaded with water. Next, cells were grown to an $\mathrm{OD}_{600}$ of $\sim 0.2$ and subsequently supplemented with either the indicated concentration of freshly DMSO-diluted flavonoid or DMSO alone (control), both at a final concentration of $0.2 \%$ DMSO. Aliquots were taken out to perform different viability stainings (PI, DHE, AnnexinV/PI), survival tests (plating, regrowth capacity) 13,55 , and/or autophagy assays (EGFP-Atg8, ALP) at indicated time points (Fig. 1a).

For the initial yeast screen, each flavonoid was supplemented at a concentration of $50 \mu \mathrm{M}$. After identification of DMC as a top cytoprotective hit, we determined the optimal dose for treatment in yeast at $100 \mu \mathrm{M}$, at least under the applied conditions. This was the lowest concentration exerting the highest rescue effect (Supplementary Fig. 2a).

One of the major causes of yeast chronological ageing might be the excessive production of acetic acid ${ }^{56}$. Consistently, yeast lifespan can be prolonged by alkalinization of the medium ${ }^{52}$. Of note, DMC treatment did not alter the $\mathrm{pH}$ of the medium (Supplementary Fig. 2f), demonstrating that the cytoprotective effects of DMC upon yeast chronological ageing are $\mathrm{pH}$-independent.

If not otherwise stated, representative ageing experiments are shown with at least six independent biological replicates aged at the same time. Experiments were performed at least three times in total with similar outcome, except during the screening procedure, where each flavonoid was tested in three to six independent samples for each ageing experiment.

Assays for cell death markers in yeast. For the initial yeast screen, PI (necrosis) and DHE (superoxide anione $\mathrm{O}_{2}^{-}$production) staining was performed as follows: $\sim 1 \times 10^{7}$ cells were harvested by centrifugation for $5 \mathrm{~min}$ at $2,700 \times g$ and stained for 5 min with propidium iodide $(100 \mathrm{ng} / \mathrm{ml}$ in PBS $\mathrm{pH}$ 7.4) or dihydroethidium $(2.5 \mu \mathrm{g} / \mathrm{ml}$ in PBS pH 7.4), respectively. Cells were pelleted again for $5 \mathrm{~min}$ at $2700 \times g$ and resuspended in PBS. Relative fluorescence units were determined using a fluorescence reader (Tecan, GeniusPRO) and normalised to the $\mathrm{OD}_{600}$ of each sample. Then, the area under the curve (AUC) for all monitored days throughout chronological ageing was calculated and a $Z$-score computed for the results with PI and DHE, respectively. The results obtained in these highthroughput assays positively correlate with the corresponding low scale experiments (Supplementary Fig. $1 \mathrm{a}-\mathrm{c}$ ). Of note, an unstained sample of each well was tested at day 1 of chronological ageing to take account for possible intrinsic fluorescence properties of any given flavonoid that might interfere with our fluorescence-based assays. For the viability assay using outgrowth capacity, which has been previously used to determine ageing-associated viability 41,57 , aliquots ( 9 $\mu \mathrm{l})$ were taken at day 3 of chronological ageing to inoculate $171 \mu \mathrm{l}$ fresh SCD media in 96-well-plates. Cultures (total $180 \mu \mathrm{l}$ ) were grown at $28^{\circ} \mathrm{C}, 1000 \mathrm{rpm}$ (motor speed) and $\mathrm{OD}_{600}$ was measured at the point of inoculation and $10 \mathrm{~h}$ thereafter using a plate reader (Tecan, GeniusPRO). Outgrowth was defined as the difference between the measured $\mathrm{OD}_{600}$ at the time of inoculation and after $10 \mathrm{~h}$ of growth $\left[\mathrm{OD}_{600(10 \mathrm{~h})}-\mathrm{OD}_{600(\text { inoculation })}\right]$, and then normalised to the DMSO-treated control; finally, a $Z$-score was computed. As in the other high-throughput assays (see above), a positive correlation between outgrowth capacity and viability was established (Supplementary Fig. 1b). 
Assays for apoptosis/necrosis (AnnexinV/PI co-staining) upon DMC treatment were quantified by flow cytometry (BD LSRII Fortessa, BD Biosciences). Briefly, $\sim 1 \times 10^{7}$ cells were harvested by centrifugation for $5 \mathrm{~min}$ at $2700 \times g$ and washed once with water and once with buffer $\mathrm{B}+\mathrm{S}(35 \mathrm{mM}$ potassium phosphate buffer, $\mathrm{pH} 6.8,0.5 \mathrm{mM} \mathrm{MgCl} 2,1.2 \mathrm{M}$ sorbitol). To obtain spheroplasts, cells were resuspended in $330 \mu$ l buffer B + S, $15 \mu$ glucuronidase/arylsulfatase (SigmaAldrich, [BGALA-RO]) and $3 \mu$ lyticase (1000 U/ml, Sigma-Aldrich [L2524]) were added, and cells were incubated at $28^{\circ} \mathrm{C}$ for $30 \mathrm{~min}$. Spheroplasts were pelleted at $500 \mathrm{~g}$ for $2 \mathrm{~min}$ and carefully washed once with buffer $\mathrm{B}+0.6 \mathrm{M}$ sorbitol. Then spheroplasts were resuspended in $30 \mu \mathrm{l}$ incubation buffer (10 mM HEPES pH 7.4, $140 \mathrm{mM} \mathrm{NaCl}, 5 \mathrm{mM} \mathrm{CaCl}_{2}, 0.6 \mathrm{M}$ sorbitol) and stained for $20 \mathrm{~min}$ by addition of

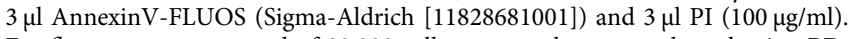
For flow cytometry, a total of 30,000 cells per sample were evaluated using BD FACSDiva software (BD Biosciences). ROS accumulating cells were quantified evaluated by DHE staining (see above) and subsequent flow cytometry and analysis of 30,000 cells. For clonogenic survival plating, cell counts of DMC-treated cultures and controls were measured using a CASY cell counter (Schärfe System GmbH), and 500 cells were plated on YEPD agar plates and incubated for two days at $28^{\circ} \mathrm{C}$ to allow colony formation. The colony-forming units (CFUs) were analysed using an automated colony counter (LemnaTech). For each strain, the CFUs determined for the control cultures were set to $100 \%$, and the survival of the respective DMCtreated cultures was calculated relative to the corresponding control culture.

\section{Plasmid construction and yeast knockout generation. Single and double-mutant} strains were generated according to a reported method by either employing a genespecific URA3-knockout cassette, amplified by PCR using pUG72 as a template ${ }^{58}$ or using the natNT2 or hphNT1 cassettes of pFA6a-natNT2 and pFA6a-hphNT1, respectively ${ }^{53}$. Correct gene deletion was verified by PCR with corresponding control primers and further checked for consistent auxotrophies. All primers used are listed in Supplementary Table 4. The plasmid [pESC-His-6(HA)] was constructed by digestion with $\mathrm{SacI} / \mathrm{ClaI}$ and ligation with the 6(HA) fragment amplified from pYM17 using primers ClaI_6HA_f and SacI_6HA_r. GLN3 was amplified from genomic DNA from BY4742, using primers GLN3_F (NotI) and GLN3_R (ClaI) and cloned into the resulting vector using NotI/ClaI restriction sites (see Supplementary Table 5). Notably, at least three different clones of each generated mutant were tested for similar response to PI staining during ageing to rule out clonogenic variation.

Yeast autophagy measurements. Autophagy was examined according to published methods by determining either GFP liberation via immunoblot analysis (see Immunoblotting section) or vacuolar localization of Atg8 through fluorescence microscopy in cells expressing an EGFP-Atg8 fusion protein ${ }^{52}$. In addition, ALP activity ${ }^{16}$ was assessed using corresponding Pho8 $\Delta$ N60 cells transformed with and selected for stable insertion of pTN9 HindIII fragment ${ }^{59}: \sim 3 \times 10^{7}$ cells were harvested by centrifugation at $2700 \times g$ for 5 min and washed once with $500 \mu \mathrm{H}_{2} \mathrm{O}$. Cells were resuspended in $200 \mu \mathrm{l}$ ice-cold Assay buffer $(250 \mathrm{mM} \mathrm{Tris} / \mathrm{HCl} \mathrm{pH} \mathrm{9.0,}$ $10 \mathrm{mM} \mathrm{MgSO}_{4}, 10 \mu \mathrm{M} \mathrm{ZnSO}$ ) and transferred to a pre-cooled reaction tube with $100 \mu \mathrm{l}$ acid-washed glass beads. Cells were disrupted in a beadmill (Mini-Beadbeater) in a pre-cooled metal reaction tube rack for $3 \times 45 \mathrm{~s}$ with $30 \mathrm{~s}$ intervals in between. Cell debris was removed by centrifugation at $10,000 \times g$ at $4{ }^{\circ} \mathrm{C}$ for $10 \mathrm{~min}$ and the supernatant carefully transferred to fresh pre-cooled tubes. Protein concentration was determined using a Bradford protein assay (Bio-Rad [5000006]). Cell extracts corresponding to $1-1.5 \mu \mathrm{g}$ protein were added to a final volume of 550 $\mu \mathrm{l}$ assay buffer (room temperature) and the reaction was started by addition of $50 \mu \mathrm{l}$ $\alpha$-naphtylphosphate (55 $\mathrm{mM}$ in assay buffer, $\mathrm{pH} 9.0$ ). After $20 \mathrm{~min}$ at room temperature, the reaction was stopped by addition of $200 \mu \mathrm{l}$ stopping buffer $(2 \mathrm{M}$ glycin/ $\mathrm{NaOH} \mathrm{pH} \mathrm{11.0).} \mathrm{Two} \mathrm{hundred} \mathrm{microlitres} \mathrm{of} \mathrm{the} \mathrm{reaction} \mathrm{mix} \mathrm{was} \mathrm{measured}$ in black 96-well plates in a platereader (Tecan, GeniusPRO) at ex: $345 \mathrm{~nm}$, em: 472 $\mathrm{nm}$. To correct for intrinsic (background) ALP activity, control cultures (without pTN9) were simultaneously processed and ALP activity subtracted.

Yeast TORC1 activity. Cells were grown to an $\mathrm{OD}_{600}$ of 0.2 and treated with either $100 \mu \mathrm{M}$ DMC or $40 \mathrm{nM}$ rapamycin for $6 \mathrm{~h}$. Three $\mathrm{OD}_{600}$ units were harvested and proteins extracted (see Immunoblotting section). Rps6 phosphorylation at serine 232 and 233 was detected with a phospho-S6 ribosomal protein (Ser235/236) antibody ${ }^{60}$ (Cell Signaling [\#2211 S], rabbit, 1:1000) and phosphorylation levels normalised to GAPDH. As controls, stationary phase cultures of wild type and rps6aS232A,S233A $\Delta r p s 6 b$ strains (kind gift from Dr. Tarek Moustafa) were refed with fresh SCD medium for $1 \mathrm{~h}$ before harvest.

Yeast MEP2 promoter activation. MEP2 promoter-dependent lacZ expression was determined using a beta-galactosidase assay. At the indicated time points, 1.5 $\mathrm{OD}_{600}$ units were harvested, washed once with water and lysed for $30 \mathrm{~s}$ in $380 \mu \mathrm{l} \mathrm{Z}$ buffer $\left(100 \mathrm{mM}\right.$ sodium phosphate buffer $\mathrm{pH} 7.0,10 \mathrm{mM} \mathrm{KCl}, 1 \mathrm{mM} \mathrm{MgSO}_{4}, 40$ $\mathrm{mM} 2$-mecaptoethanol) with $50 \mu \mathrm{l} 0.1 \% \mathrm{SDS}$ and $50 \mu \mathrm{CHCl}_{3}$ using a beadmill (Mini-Beadbeater). After addition of $50 \mu \mathrm{l} 4 \mathrm{mg} / \mathrm{ml} \mathrm{o}$-nitrophenylgalactoside (Serva), suspensions were incubated at room temperature and stopped with $125 \mu \mathrm{l}$ $1 \mathrm{M} \mathrm{Na}_{2} \mathrm{CO}_{3}$ when the suspensions turned yellow. Samples were centrifuged for 5 $\mathrm{min}$ at $2700 \times g$ and absorption of the supernatant was measured at $450 \mathrm{~nm}$. To assess the fraction of live cells, aliquots of the cultures were stained with PI and cell death measured by flow cytometry. Miller units/fraction alive were calculated using the formula [1000x A $\left.\mathrm{A}_{450}\right] /\left[\right.$ volume in $\mathrm{ml}^{*} \mathrm{OD}_{600}{ }^{*}$ incubation time in $\min ^{*}$ fraction PI-negative cells].

Determination of yeast metabolomic changes. Yeast cells cultured in deep well plates were treated with $100 \mu \mathrm{M}$ DMC (or $0.2 \%$ DMSO as control) for $72 \mathrm{~h}$. For each replicate, $5 \mathrm{ml}$ culture were rapidly harvested by microfiltration using $0.45 \mu \mathrm{m}$ PVDF-filters, immediately washed with $10 \mathrm{ml}$ ultrapure water, snap-frozen in liquid nitrogen, and kept at $-80{ }^{\circ} \mathrm{C}$ prior to extraction. Boiling ethanol (BE) extraction was performed with $2.5 \mathrm{ml}$ pre-heated BE buffer $(75 \% \mathrm{v} / \mathrm{v}$ ethanol, 15 $\mathrm{mM}$ ammonium acetate $\mathrm{pH}$ 7.5) for $2 \mathrm{~min}$ at $96^{\circ} \mathrm{C}$ in a water bath with short vortexing every $30 \mathrm{~s}$. After extraction, the cells' remnants were pelleted by centrifugation for $3 \mathrm{~min}$ at $2500 \times \mathrm{g}$ at $-20^{\circ} \mathrm{C}$, the supernatant was concentrated by nitrogen evaporation and dry-frozen. Samples were dissolved in $100 \mu \mathrm{LCMS}-\mathrm{H}_{2} \mathrm{O}$, centrifuged at $17,000 \times g$ for $5 \mathrm{~min}$ and the supernatant used for LC/MS.

All samples were measured with a LC/MS system from Thermo Fisher ScientificTM. A Dionex Ultimate 3000 HPLC setup equipped with an Atlantis T3 C18 analytical column (Waters, USA) was used for compound separation prior to mass spectrometric detection with an ExactiveTM Orbitrap system. A reversedphase ion-pairing HPLC method was used for metabolite separation (adapted from ref. ${ }^{61}$ ). Tributylamine was used as ion-pairing agent. A 40 min gradient was applied and 2-propanol and an aqueous phase (5\% methanol (v/v), $10 \mathrm{mM}$ tributylamine, $15 \mathrm{mM}$ acetic acid, $\mathrm{pH}$ 4.95) were used as eluent $\mathrm{A}$ and $\mathrm{B}$, respectively (Supplementary Table 6). The injection volume was $10 \mu$ per sample and an injection loop of $20 \mu \mathrm{l}$ was used.

Negative ionisation of metabolites was carried out via heated electrospray ionization (HESI) prior mass spectrometric analysis. For the online detection of the analytes a full scan of all masses between 70 and $1100 \mathrm{~m} / z$ with a resolution (R) of $50,000($ at $m / z 200)$ was used.

LC/MS-data acquisition was conducted with Xcalibur software (version 2.2 SP1, Thermo Fisher Scientific (Waltham, USA)), Raw data were converted into mzXML by msConvert (ProteoWizard Toolkit v3.0.5), and metabolites were targetedsearched using the in-house developed tool PeakScout ${ }^{62}$. Pure analytes were run on the same system to obtain exact reference retention times and fragmentation spectra. Raw data was further assessed with Microsoft Excel 2010. For metabolite clustering, metabolite areas were normalised to the cumulative signal of all metabolite areas for each day (the mean signal of each metabolite across all samples was set to 1 ) and $\log 2$ transformed. PCA analyses were performed using the tool Genesis 1.7.7 (Bioinformatics, Technical University of Graz). The complete data set is available in Supplementary Data File 1.

Determination of yeast proteome using SILAC. SILAC (stable isotope labeling by/with amino acids in cell culture) experiments were performed following previously published protocols ${ }^{17}$. Briefly, proteins from labeled yeast cells (Lys $0+$ Arg0 or Lys $4+$ Arg10) treated with $100 \mu \mathrm{M}$ DMC for $24 \mathrm{~h}$ or $72 \mathrm{~h}$ were extracted by glass bead disruption in buffer $\mathrm{P}(50 \mathrm{mM}$ Tris/ $\mathrm{HCl} \mathrm{pH} \mathrm{7.4,1 \%}$ Triton X-100, $150 \mathrm{mM} \mathrm{NaCl}, 1 \mathrm{mM}$ EDTA) containing complete ${ }^{\circledast}$ protease inhibitor cocktail (Roche), $1 \mathrm{mM}$ PMSF (Sigma) and HDAC inhibitors trichostatin A $(2 \mu \mathrm{M}$, Sigma) and nicotinamide (30 $\mathrm{mM}$, Sigma). Protein concentration was determined using Bio-Rad protein assay (Bio-Rad) and $500 \mu \mathrm{g}$ from each heavy and light extracts were mixed and stored at $-80^{\circ} \mathrm{C}$ prior to MS measurement. For MS sample preparation, probes were reduced with $1 \mathrm{mM}$ DTT (Sigma-Aldrich) and alkylated using $5.5 \mathrm{mM}$ iodoacetamide (Sigma-Aldrich). Proteins were separated by SDSPAGE and digested in gel using trypsin (Promega) at $37^{\circ} \mathrm{C}$ over night and the resulting peptide mixtures were processed on STAGE tips ${ }^{63}$.

Mass spectrometric measurements were performed on a LTQ Orbitrap XL mass spectrometer (Thermo Fisher Scientific, Bremen, Germany) coupled to an Agilent 1200 nanoflow-HPLC (Agilent Technologies GmbH, Waldbronn, Germany). HPLC-column tips (fused silica) with $75 \mu \mathrm{m}$ inner diameter (New Objective, Woburn, MA, USA) were self-packed with Reprosil-Pur 120 ODS-3 (Dr. Maisch, Ammerbuch, Germany) to a length of $20 \mathrm{~cm}$. Samples were applied directly onto the column without pre-column. A gradient of A $[0.5 \%$ acetic acid (high purity, LGC Promochem, Wesel, Germany) in water (HPLC gradient grade, Mallinckrodt Baker B.V., Deventer, Netherlands)] and B [0.5\% acetic acid in 80\% ACN (LC-MS grade, Wako, Germany) in water] with increasing organic proportion was used for peptide separation (loading of sample with $2 \% \mathrm{~B}$; separation ramp: from $10 \%$ to $30 \%$ B within $80 \mathrm{~min}$ ). The flow rate was $250 \mathrm{nl} / \mathrm{min}$ and for sample application $500 \mathrm{nl} / \mathrm{min}$. The mass spectrometer was operated in the data-dependent mode and switched automatically between MS (max. of $1 \times 10^{6}$ ions) and MS/MS. Each MS scan was followed by a maximum of five MS/MS scans in the linear ion trap using normalised collision energy of $35 \%$ and a target value of 5000 . Parent ions with a charge state from $z=1$ and unassigned charge states were excluded for fragmentation. The mass range for MS was $m / z=370$ to 2000 . The resolution was set to 60,000 . Mass-spectrometric parameters were as follows: spray voltage $2.3 \mathrm{kV}$; no sheath and auxiliary gas flow; ion-transfer tube temperature $125^{\circ} \mathrm{C}$

The MS raw data files were uploaded into the MaxQuant software version 1.4.1.2 $2^{64}$, which performs peak detection, label-free quantification, and generates peak lists of mass error corrected peptides using the following parameters: carbamidomethylcysteine was set as fixed modification, methionine oxidation and 
protein amino-terminal acetylation were set as variable modifications. Three miss cleavages were allowed, enzyme specificity was trypsin/P, and the MS/MS tolerance was set to $0.5 \mathrm{Da}$. Peak lists were searched by Andromeda for peptide identification using a Uniprot Saccharomyces cerevisiae database containing common contaminants such as keratins and enzymes used for in-gel digestion. Peptide lists were further used by MaxQuant to identify and relatively quantify proteins using the following parameters: peptide, and protein false discovery rates were set to 0.01 , maximum peptide posterior error probability (PEP) was set to 1 , minimum peptide length was set to 7, the PEP was based on Andromeda score, minimum number peptides for identification and quantitation of proteins was set to one and must be unique, and identified proteins were re-quantified.

For enrichment analysis, the DAVID Bioinformatics Resources 6.7 software, NIAID/NIH [https://david-d.ncifcrf.gov/], was used employing the following settings: functional annotation clustering (GOTERM_BP_FAT), medium stringency, Kappa similarity (term overlap: 3 , similarity threshold: 0.5 ), classification (initial group membership: 3 , final group membership: 3 , multiple linkage threshold: 0.5), enrichment Threshold (EASE: 1.0) The reference proteome was set to all measured proteins at a given timepoint. For $P$-value calculation, Benjamini correction was used. The complete data set is available in Supplementary Data File 2.

The mass spectrometry proteomics data have been deposited to the ProteomeXchange Consortium via the PRIDE ${ }^{65}$ partner repository with the dataset identifier PXD012108 and the project name: "The flavonoid 4,4'dimethoxychalcone promotes autophagy-dependent longevity across species".

C. elegans strains and maintenance. We followed standard nematode culture conditions $s^{66}$. Nematode rearing temperature was kept at $20^{\circ} \mathrm{C}$, and animals were maintained at $20^{\circ} \mathrm{C}$ on Nematode Growth Media (NGM) agar supplemented with Escherichia coli (OP50 or transformed HT115). We employed the N2 (wild type) and MH4799:elt-1(ku491) IV strains for lifespan assays and the VK1093:vkEx1093 $\left[\mathrm{P}_{\text {nhx-2 }}:: \mathrm{mCherry}:\right.$ lgg-1] strain for autophagy measurements.

C. elegans lifespan assays. DMC-treatment of C. elegans was carried out on NGM agar plates continuously during the entire lifetime starting as L1 larvae, unless otherwise specified. All agar plates were prepared from the same batch of NGM agar and seeded with dried bacteria (OP50), which were previously UV-killed to avoid interference with the xenobiotic-metabolising activity of Escherichia coli. DMC $(41.6 \mu \mathrm{M})$ or an equivalent volume of the solvent DMSO was spotted on dried bacteria in treatment and control plates, respectively. Spotted plates were dried overnight at room temperature prior to use. The procedure was repeated each time worms were transferred to fresh plates. For RNAi lifespan experiments, RNAi feeding started at L4 larval stage. Worms were placed on NGM plates containing 0.5-1 mM IPTG and seeded with HT115 bacteria transformed with either the pL4440 vector or the test RNAi plasmid. The dsRNA-transformed bacteria atg- 5 (Y71G12B.12), bec-1 (T19E7.3) and elt-1 (W09C2.1) were derived from the Ahringer C. elegans RNAi library ${ }^{67}$. All dsRNA bacterial clones were used at a concentration of 0.9 OD diluted with empty vector-harbouring bacteria, according to previous studies ${ }^{68}$.

Survival analysis started from hatching, unless otherwise specified, and was carried out at $20^{\circ} \mathrm{C}$ using synchronous populations of $60 / 80$ animals per condition. Animals were scored as dead or alive and transferred every day on fresh plates during the fertile period, and then every other day or every 3 days until death. Worms were considered dead when they stopped pharyngeal pumping and responding to touch. Worms that died because of internal bagging, desiccation due to crawling on the edge of the plates, or gonad extrusion were scored as censored. These animals were included in lifespan analyses up to the point of censorship. Note that due to the rather high biological variance associated with ageing experiments, replicates of survival assays are shown as separate experiments; for details, see Supplementary Table 2.

\section{C. elegans autophagy measurements. To quantify autophagy induction, the}

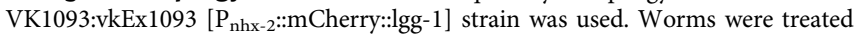
with $41.6 \mu \mathrm{M}$ DMC from the L1 stage for $48 \mathrm{~h}$. For silencing experiments, worms were transferred as $\mathrm{L} 4$ on the respective plates (control and corresponding RNAi \pm $\mathrm{DMC}$ ). After $24 \mathrm{~h}$ of silencing the (adult) worms were acquired using a fluorescence microscope.

Using ImageJ, [http://imagej.nih.gov/ij/], the pictured worms were manually selected and the number of foci counted, using the automated function in Image] "Analyze Particles" on the adjusted images. To avoid artifacts, all the pictures were acquired and adjusted using the same settings.

D. melanogaster strains and media. We used Drosophila melanogaster $\mathrm{w}^{1118}$ or Atg $7^{-1-}$ flies. The latter were generated by crossing Atg $7^{\mathrm{d} 77 /+}$ virgins with Atg $7^{\mathrm{d} 14 /+}$ male flies ${ }^{69}$, both isogenised to $\mathrm{w}^{1118}$. Fly experiments were performed using two different media: (i) CSY, containing $1 \%$ agar (BD) $1 \%$ Bacto ${ }^{\text {ma }}$ yeast extract (BD), $5 \%$ sucrose (Roth), $0.8 \%$ cornmeal, $0.03 \%$ (v/v) propionic acid and $0.3 \%(\mathrm{v} / \mathrm{v})$ 4-hydroxybenzoate solution (Sigma-Aldrich, $100 \mathrm{~g} / \mathrm{l}$ in 100\% ethanol), or (ii) Bloomington cornmeal-molasses medium, containing $0.8 \%$ agar, $0.75 \%$ dry baker's yeast, $0.83 \%$ soy meal, $8.5 \%$ sugar beet syrup, $6.7 \%$ corn meal, $0.0525 \%(\mathrm{v} /$ v) propionic acid and $0.42 \%$-hydroxybenzoate solution ( $310 \mathrm{~g} / \mathrm{l}$ in $100 \%$ ethanol). For both media, propionic acid and 4-hydroxybenzoate were added when the food had cooled down to $60^{\circ} \mathrm{C}$. DMC was added at $40^{\circ} \mathrm{C}$ at a final concentration of 200 $\mu \mathrm{M}$ and a DMSO concentration of $0.1 \%$. Food was stored at $4{ }^{\circ} \mathrm{C}$ for no longer than 1 week.

D. melanogaster lifespan analyses. Flies were reared in standard wide plastic vials with plugs and hatched for three days until they were collected, allowed to mate for $24 \mathrm{~h}$ and then sexed under $\mathrm{CO}_{2}$ anaesthesia and sorted into portions of 20 flies with a maximal, total anaesthesia time of $6 \mathrm{~min}$. For brain immunofluorescence, flies were anesthetised for no longer than $2 \mathrm{~min}$. On CSY, flies were transferred to fresh food three times a week, on Bloomington cornmeal-molasses medium every other day. Accidentally escaped flies were censored. All ageing experiments were carried out in climate chambers at $25^{\circ} \mathrm{C}$ and $70 \%$ humidity with a $12 \mathrm{~h} / 12 \mathrm{~h}$ light/dark cycle. Note that due to the rather high biological variance associated with ageing experiments, replicates of survival assays are shown as separate experiments; for details, see Supplementary Table 2.

Fly food consumption assay. Feeding behaviour was determined by capillary feeding assays. Briefly, plastic fly vials were filled with $3 \mathrm{ml} 1 \%$ agar and three smal air holes were introduced into the vial wall with a hot syringe. Vials were sealed with Parafilm (Bemis NA) and rubber caps, containing two additional air holes for ventilation and two holes for $5 \mu$ glass capillaries (Hirschmann). Four female flies per vial were accustomed to the feeding procedure for 2 days. Then, feeding of the test food (2.5\% yeast extract, $2.5 \%$ sucrose and $200 \mu \mathrm{M}$ DMC or respective amount of DMSO) was monitored over three consecutive $10-15 \mathrm{~h}$ intervals. Five independent vials per condition were analysed and feeding behaviour expressed as $\mu \mathrm{l}$ food $^{\star} \mathrm{fly}^{-1 *} 12 \mathrm{~h}^{-1}$

Fly fecundity assay. Flies (1-4 days old) were transferred to new vials and allowed to mate for $48 \mathrm{~h}$ before sorting them into portions of 10 female animals under $\mathrm{CO}_{2}$ anaesthesia. Flies were aged on Bloomington food containing $200 \mu \mathrm{M}$ DMC or control food. At the indicated time points, flies were transferred to fresh food for $16 \mathrm{~h}$ and subsequently first instar larvae and eggs were counted. In total, six independent vials per condition were analysed.

Fly autophagy measurements. $\mathrm{w}^{1118}$ flies were reared on Bloomington cornmealmolasses medium, containing $0.47 \%$ agar, $0.75 \%$ dry baker's yeast, $0.83 \%$ soy meal, $8.5 \%$ sugar beet syrup, $6.7 \%$ corn meal, $0.0525 \%$ (v/v) propionic acid and $0.42 \% 4$ hydroxybenzoate solution $(310 \mathrm{~g} / \mathrm{l}$ in $100 \%$ ethanol) and allowed to hatch for 2 days. Then flies were transferred to new vials and allowed to mate for $24 \mathrm{~h}$. Portions of 20 female flies were sorted under $\mathrm{CO}_{2}$ anaesthesia with a maximal anaesthesia of $2 \mathrm{~min}$ and transferred to small vials containing control food $(0.1 \%$ DMSO) or food supplemented with $200 \mu \mathrm{M}$ DMC, both containing $1 \%$ agar. Flies were transferred to fresh vials every other day and aged for 3 (young) or 30 days (old) at $25^{\circ} \mathrm{C}$ and $70 \%$ humidity with a $12 \mathrm{~h} / 12 \mathrm{~h}$ light/dark cycle.

Brains from 30-days old female flies were dissected and collected in ice cold haemolymph-like saline (HL-3) solution. Following fixation in phosphate buffered saline containing $0.7 \%$ Triton X-100 (PBS-T) and $4 \%$ paraformaldehyde for $30 \mathrm{~min}$ and several washing steps in PBS-T, unspecific antibody binding sites were blocked with $10 \%$ normal goat serum. Brains were incubated with a polyclonal anti-Ref(2)P (Rabbit, gift from Dr. Gabor Juhasz, 1:8000) antibody for 48 h, washed six times, and subsequently incubated with the corresponding Alexa 488-linked secondary antibody (Rabbit, Invitrogen, [\#A11034], 1:500) for another 16h. 11-12 brains of each condition were stored in VectaShield (Vector Laboratories LTD.,

Peterborough, United Kingdom) in glass-bottom petri dishes at $4{ }^{\circ} \mathrm{C}$ and scanned by confocal microscopy within $2-4$ weeks.

Spatial image data was generated using a Leica SP5 confocal microscope with spectral detection (Leica Microsystems, Inc.) and a HCX IRAPO L 25x/0.95 NA water immersion objective. Z-stacks were acquired using a resonant scanner $(8000$ $\mathrm{Hz}, 8 \times$ line averaging, $1024 \times 1024$ pixels). Alexa 488 fluorescence was excited at $488 \mathrm{~nm}$ and emission detected at $500-550 \mathrm{~nm}$. Image noise was reduced by image deconvolution (Huygens Pro, SVI B.V.; optimised maximum-likelihood estimation approach; five iterations). Processed z-stacks were projected using the maximumintensity projection method. Image analysis of projections was performed using ImageJ software, [http://rsbweb.nih.gov/ij/]. The central brain region of 11-12 brains per condition was selected manually with the free-hand tool, and the average grey value related to the selected area of each brain was measured.

Cell culture conditions and cell lines. Culture media and supplements for cell culture were purchased from Gibco-Invitrogen (Carlsbad, CA, USA) and plasticware from Corning (Corning, NY, USA). Human osteosarcoma U2OS cells (cell line was purchased from ATCC [HTB-96]) and their GFP-LC3-expressing derivatives were cultured in DMEM medium containing $10 \%$ foetal bovine serum, 100 $\mathrm{mg} / \mathrm{l}$ sodium pyruvate, $10 \mathrm{mM}$ HEPES buffer, 100 units $/ \mathrm{ml}$ penicillin $\mathrm{G}$ sodium and $100 \mu \mathrm{g} / \mathrm{ml}$ streptomycin sulphate $\left(37^{\circ} \mathrm{C}, 5 \% \mathrm{CO}_{2}\right)$. Human hepatocellular carcinoma HepG2 cells (cell line was purchased from ATCC [HB8065]) were cultured in EMEM medium supplemented with $10 \%$ fetal bovine serum, $100 \mathrm{mg} / \mathrm{l}$ sodium pyruvate, $10 \mathrm{mM}$ HEPES buffer, $100 \mathrm{units} / \mathrm{ml}$ penicillin G sodium and 100 
$\mu \mathrm{g} / \mathrm{ml}$ streptomycin sulphate. Human colorectal cancer HCT116 cells (cell line was purchased from ATCC [CCL-247]) were cultured in McCoy's medium enriched with $10 \%$ fetal bovine serum, $100 \mathrm{mg} / \mathrm{l}$ sodium pyruvate, $10 \mathrm{mM}$ HEPES buffer, 100 units $/ \mathrm{ml}$ penicillin $\mathrm{G}$ sodium and $100 \mu \mathrm{g} / \mathrm{ml}$ streptomycin sulfate. For autophagy induction, cells were treated for $8 \mathrm{~h}$ with $50 \mu \mathrm{M}$ DMC (Sigma Aldrich [S617237]) in presence or absence of $50 \mu \mathrm{M}$ chloroquine (Sigma Aldrich [C6628]) to properly assess autophagic flux.

Cell culture autophagy measurements by automated microscopy. GFP-LC3 U2OS cells were generated by transfection of U2OS cells with pEGFP-LC3 plasmid and were maintained by selection with Neomycin. GFP-LC3 HCT116 cells were generated by transfection with lentiviral GFP-LC3 construct (Millipore [1710193]). U2OS or HCT116 cells stably expressing GFP-LC3 were seeded in 96-well or 384-well imageing plates (Greiner) $24 \mathrm{~h}$ before stimulation. Cells were treated with DMC for $8 \mathrm{~h}$, in presence or absence of Chloroquine (for $2 \mathrm{~h}$ before fixation). Subsequently, cells were fixed with $4 \%$ PFA and counterstained with $10 \mu \mathrm{M}$ Hoechst 33342. Images were acquired using a BD pathway 855 automated microscope (BD Imageing Systems, San José,USA) equipped with a 40X objective (Olympus, Center Valley, USA) coupled to a robotised Twister II plate handler (Caliper Life Sciences, Hopkinton, USA). Images were analysed for the presence of GFP-LC3 dots in the cytoplasm by means of the BD Attovision software (BD Imageing Systems). Cell surfaces were segmented and divided into cytoplasmic and nuclear regions according to manufacturer standard proceedings. RB $2 \times 2$ and Marr-Hildreth algorithms were used to recognise cytoplasmic GFP-LC3 positive dots. For siRNA analyses, U2OS cells stably expressing GFP-LC3 were transfected with scramble siRNA (siCtr) or siRNAs targeting GATA1 (siGATA1), GATA2 (siGATA2), GATA3 (siGATA3), GATA4 (siGATA4), GATA5 (siGATA5), GATA6 (siGATA6), TRPS1 (siTRPS1) and Atg5 (siAtg5) (3 individual siRNA sequences per each gene, see Table 5) for $48 \mathrm{~h}$, followed by $50 \mu \mathrm{M} \mathrm{DMC} \mathrm{(or} 10 \mu \mathrm{M}$ rapamycin) or being kept in control condition for additional $6 \mathrm{~h}$. Thereafter, cells were fixed and autophagy measured as described above. For quantification, the mean value and SEM from the data of all three individual siRNA sequences were calculated. Knockdown efficiency was verified by western blot analysis (Supplementary Figure 13).

Yeast-like chronological senescence assay. The assay was performed as described in Leontieva et $\mathrm{al}^{70}$. In brief, 80,000 cells were seeded into 96 -well plates and left untreated or treated with $50 \mu \mathrm{M}$ DMC. After 5 days, dead cells and conditioning media were removed, cells were trypsinised and a $10 \%$ aliquot was plated in fresh medium-filled six-well plates. After 1 week, clones were marked trough crystal violet staining and counted.

Determination of S6K1 phosphorylation. U20S cells were treated for $6 \mathrm{~h}$ with two different doses of DMC or rapamycin $(1 \mu \mathrm{M})$. mTORC1 activity was assessed by immunoblotting and evaluated as levels of phosphorylation of its target ribosomal protein S6 kinase beta-1 (S6K1/P70S6K1) on threonine 389 using a phosphospecific antibody (Cell Signalling Technology, [\#9205]). Rapamycin was used as a positive control of mTORC1 inhibition.

Plant verification and extraction. Angelica keiskei koidzumi plants were purchased from a local retailer. The plants displayed the morphological characteristics for the species, including pinnate leaves, serrate, 3-parted leaflets, long petioles with sheathing and the characteristic colour of its sap. In addition, plant DNA extracted from snap-frozen leaves was genotypically verified. Therefore, nuclear rDNA (internal transcribed spacer 1, 5.8S ribosomal RNA gene and internal transcribed spacer 2) was amplified with primers ITS4 and ITS5 (Supplementary Table 5) and the product sequenced using the same primers (MWG Biotech, Ebersberg, Germany). Comparison to GenBank accession no. GU395158.1 confirmed the genotype for Angelica keiskei koidzumi.

For detection of DMC in Angelica keiskei koidzumi, fresh plant material ( $2 \mathrm{~g}$ of roots and stipes/leaves, respectively) was harvested, cut in $5 \times 5 \mathrm{~mm}$ pieces, snapfrozen and pulverised with pre-cooled steel beads in a bead mill (mini-beadbeater, Biospec Products). Plant powder was dry-frozen and kept at $-80^{\circ} \mathrm{C}$ for at least 24 $\mathrm{h}$ prior to extraction (for extraction procedure, refer to "Extractions and measurements of DMC").

Mouse husbandry and serum preparation. Male C57BL/6 mice were purchased from Janvier Labs, France. Treatment of animals started at an age of 12 months. All mice were kept and treated in accordance with national and European ethical regulations (Directive 2010/63/EU) and the experiments approved by the responsible government agency (Bundesministerium für Wissenschaft, Forschung und Wirtschaft, BMWFW, Austria). Mice were fed ad libitum with regular food (pellets, Ssniff, Germany) supplemented with $0.25 \%$ DMC for 7 days. Animal physical activity, exploration, defecation, food and water intake as well as body weight was carefully monitored to ensure normal behaviour. At the end of the experiment, the animals were anaesthetised by isoflurane inhalation and sacrificed. Plasma was obtained by centrifugation at $200 \times g$ for $10 \mathrm{~min}$ at $4^{\circ} \mathrm{C}$ and kept at $-80^{\circ} \mathrm{C}$ prior to extraction.
For mouse autophagy measurements, 6-week-old male wild type C57BL/6 or Atg $4 b^{-1-}$ mice (Harlan, France) were used. Animal experiments were in compliance with the EU Directive 63/2010 and protocol APAFIS \#105112017070511526660 v2 was approved by the Ethical Committee of the CRC (CEEA no. 5, registered at the French Ministry of Research). Atg $4 b^{-1-}$ mice were kindly provided by Dr. Carlos Lopez-Otin (Oviedo University, Spain).

Mice were housed in a temperature-controlled environment with $12 \mathrm{~h}$ light/ dark cycles and received food and water ad libitum. Mice were injected intraperitoneally with a single $100 \mathrm{mg} / \mathrm{kg}$ dose of DMC, dissolved in $50 \mu \mathrm{DMSO}$, and $30 \mathrm{mg} / \mathrm{kg}$ of Leupeptin ( $2 \mathrm{~h}$ prior to sacrifice) and $6 \mathrm{~h}$ later were sacrificed. Tissues were snap-frozen in liquid nitrogen and homogenised (two cycles of $20 \mathrm{~s}$ at $5500 \mathrm{rpm}$ [motor speed]) using Precellys 24 tissue homogenator (Bertin Technologies, Montigny-le-Bretonneux, France) in a $20 \mathrm{mM}$ Tris buffer ( $\mathrm{pH}$ 7.4) containing $150 \mathrm{mM} \mathrm{NaCl}, 1 \%$ Triton X-100, $10 \mathrm{mM}$ EDTA and Complete ${ }^{\circledast}$ protease inhibitor cocktail (Roche Applied Science, Penzberg, Germany). Tissue extracts were then centrifuged at $12,000 \times g$ at $4{ }^{\circ} \mathrm{C}$ and supernatants were collected. Protein concentration in the supernatants was evaluated by the bicinchoninic acid technique (BCA protein assay kit, Pierce Biotechnology, Rockford, USA).

For prolonged ischemia in vivo, 3-month-old male C57BL/6J wild type or Atg7 cardiac-specific knockout (Atg7cKO) mice were obtained from the Jackson Laboratory. All mice were kept and treated in accordance with national ethical regulations (IACUC protocol \#17022) and the experiments approved by the responsible institutional agency (Rutgers-New Jersey Medical School's Institutional Animal Care and Use Committee). Mice were housed in a temperature-controlled environment with 12-h light/dark cycles where they received food and water ad libitum. Three-month-old C57BL/6 J mice were anesthetised by intraperitoneal injection of pentobarbital sodium $(60 \mathrm{mg} / \mathrm{kg}$ ). A rodent ventilator (Minivent; Harvard Apparatus Inc.) was used with $65 \%$ oxygen. The animals were kept warm using heat lamps. Rectal temperature was monitored and maintained between 36.8 and $37.2^{\circ} \mathrm{C}$. The chest was opened by a horizontal incision at the fourth intercostal space. Ischemia was achieved by ligating the anterior descending branch of the left coronary artery (LAD) using an 8-0 prolene suture $2 \mathrm{~mm}$ below the border between the left atrium and LV. Ischemia was confirmed by ECG change (ST elevation) and colour change of myocardium. When recovered from anaesthesia, the mice were returned to their cages. They were housed in a climate-controlled environment.

Determination of metabolic changes in mice heart and liver. Animals were treated as described for mouse autophagy measurements (see "Mouse husbandry and serum preparation" section). All standard and reactives used were from SigmaAldrich except ammonium carbonate (VWR). For sample preparation of liver and heart tissue, about $30 \mathrm{mg}$ of tissues for each condition were first weighted and solubilised into $1.5 \mathrm{ml}$ polypropylene microcentrifuge tubes with ceramic beads with $1 \mathrm{ml}$ of cold lysate buffer ( $\mathrm{MeOH} /$ Water/Chloroform, 9/1/1, $-20^{\circ} \mathrm{C}$ ). They were then homogenised three times for $20 \mathrm{~s}$ at 5,500 rpm (motor speed) using Precellys 24 tissue homogenator (Bertin Technologies, Montigny-le-Bretonneux, France), followed by a centrifugation $\left(10 \mathrm{~min}\right.$ at $\left.15000 \times g, 4^{\circ} \mathrm{C}\right)$. Then upper phase of the supernatant was split in two parts: the first $270 \mu \mathrm{l}$ used for the Gas Chromatography coupled by Mass Spectrometry (GC/MS) experiment in microtubes centrifugation, the others $250 \mu \mathrm{l}$ used for the Ultra High Pressure Liquid Chromatography coupled by Mass Spectrometry (UHPLC/MS) experimentations.

Concerning the GC-MS aliquots, $150 \mu \mathrm{l}$ were transferred from the microtube centrifugation to a glass tube and evaporated. $50 \mu \mathrm{l}$ of methoxyamine $(20 \mathrm{mg} / \mathrm{ml}$ in pyridine) was added on dried extracts, then stored at room temperature in dark, for $16 \mathrm{~h}$. The day after, $80 \mu \mathrm{l}$ of MSTFA was added and final derivatization occurred at $40{ }^{\circ} \mathrm{C}$ for $30 \mathrm{~min}$. Samples were then transferred in vials and directly injected into GC-MS.

Concerning the LC-MS aliquots, the collected supernatant was evaporated in microcentrifuge tubes at $40{ }^{\circ} \mathrm{C}$ in a pneumatically-assisted concentrator (Techne DB3, Staffordshire, UK). The LC-MS dried extracts were solubilised with $450 \mu \mathrm{l}$ of MilliQ water and aliquoted in three microcentrifuge tubes $(100 \mu \mathrm{l})$ for each LC method and one microcentrifuge tube for safety.

Aliquots for analysis were transferred in LC vials and injected into LC/MS or kept at $-80^{\circ} \mathrm{C}$ until injection.

For sample preparation of polyamines (liver, heart), about $30 \mathrm{mg}$ of tissues for each condition were first weighted and solubilised into $1.5 \mathrm{ml}$ polypropylene microcentrifuge tubes, with $1 \mathrm{ml}$ of cold lysate buffer with $1 \%$ sulfosalicylic acid $\left(\mathrm{MeOH} /\right.$ water $\left.1 \% \mathrm{SSA}, 9 / 1,-20^{\circ} \mathrm{C}\right)$. They were then homogenised three times for $20 \mathrm{~s}$ at $5500 \mathrm{rpm}$ (motor speed) using Precellys 24 tissue homogenator (Bertin Technologies, Montigny-le-Bretonneux, France), followed by a centrifugation (10 $\min$ at $15,000 \mathrm{~g}, 4^{\circ} \mathrm{C}$ ). Six hundred microlitresof the upper phase of the supernatant was collected and evaporated in microcentrifuge tubes at $40^{\circ} \mathrm{C}$ in a pneumatically-assisted concentrator (Techne DB3, Staffordshire, UK). The LC-MS dried extracts were solubilised with $300 \mu \mathrm{l}$ of MilliQ water, centrifugated (10 min at $15,000 \times g, 4{ }^{\circ} \mathrm{C}$ ), and $50 \mu \mathrm{l}$ were transferred in polypropylene vial injection for LC method and the rest was transferred in microcentrifuge tube for safety. Aliquots transferred in polypropylene vials were injected into LC/MS or kept at $-80^{\circ} \mathrm{C}$ until injection.

A number of targeted and untargeted analyses were performed to obtain a comprehensive picture of DMC's metabolomic impact. Note that all MRM transitions for the below methods are available upon request. 
(1) Targeted analysis of CoAs and nucleoside phosphates by ion pairing ultrahigh performance liquid chromatography (UHPLC) coupled to a Triple Quadrupole (QQQ) mass spectrometer was performed on a RRLC 1260 system (Agilent Technologies, Waldbronn, Germany) coupled to a Triple Quadrupole 6410 (Agilent Technologies) equipped with an electrospray source operating in positive mode. The gas temperature was set to $350^{\circ} \mathrm{C}$ with a gas flow of $12 \mathrm{~L} / \mathrm{min}$. The capillary voltage was set to $3.5 \mathrm{kV}$. $10 \mu \mathrm{L}$ of sample were injected on a Column XDB-C18 $(100 \mathrm{~mm} \times 2.1 \mathrm{~mm}$ particle size $1.8 \mu \mathrm{m})$ from Agilent technologies, protected by a guard column XDB-C18 $(5 \mathrm{~mm} \times 2.1 \mathrm{~mm}$ particle size $1.8 \mu \mathrm{m})$ and heated at $40^{\circ} \mathrm{C}$ by a pelletier oven. Heat the column more than the room temperature allowed rigorous control of the column temperature. The gradient mobile phase consisted of water with $2 \mathrm{mM}$ of DBAA (A) and acetonitrile (B). The flow rate was set to $0.2 \mathrm{ml} / \mathrm{min}$, and gradient as follow: initial condition was $90 \%$ phase $\mathrm{A}$ and $10 \%$ phase $\mathrm{B}$, maintained during $4 \mathrm{~min}$. Molecules were then eluted using a gradient from $10 \%$ to $95 \%$ phase B over $3 \mathrm{~min}$. The column was washed using 95\% mobile phase B for $3 \mathrm{~min}$ and equilibrated using $10 \%$ mobile phase B for $3 \mathrm{~min}$. The autosampler was kept at $4{ }^{\circ} \mathrm{C}$. At the end of the batch of analysis, column was rinsed with $0.3 \mathrm{ml} / \mathrm{min}$ of MilliQ water (phase A) and acetonitrile (phase B) as follow: 10\% phase B during $20 \mathrm{~min}$, to $90 \%$ phase B in $20 \mathrm{~min}$, and maintained during $20 \mathrm{~min}$ before shutdown. The collision gas was nitrogen. The scan mode used was the MRM for biological samples. Peak detection and integration of the 23 analytes were performed using the Agilent Mass Hunter quantitative software (B.07.01).

(2) Widely-targeted analysis of intracellular metabolites gas chromatography (GC) coupled to a triple quadrupole (QQQ) mass spectrometer was performed on a 7890 A gas chromatography (Agilent Technologies, Waldbronn, Germany) coupled to a triple quadrupole $7000 \mathrm{C}$ (Agilent Technologies, Waldbronn, Germany) equipped with a High sensitivity electronic impact source (EI) operating in positive mode. The front inlet temperature was $250^{\circ} \mathrm{C}$, the injection was performed in splitless mode. The transfer line and the ion-source temperature were $250^{\circ} \mathrm{C}$ and $230^{\circ} \mathrm{C}$, respectively. The septum purge flow was fixed at $3 \mathrm{~mL} / \mathrm{min}$, the purge flow to split vent operated at $80 \mathrm{~mL} / \mathrm{min}$ during $1 \mathrm{~min}$ and gas saver mode was set to 15 $\mathrm{ml} / \mathrm{min}$ after $5 \mathrm{~min}$. The helium gas flowed through the column (J\&WScientificHP5MS, $30 \mathrm{~m} \times 0.25 \mathrm{~mm}$, i.d. $0.25 \mathrm{~mm}$, d.f., Agilent Technologies Inc.) at $1 \mathrm{ml} / \mathrm{min}$. Column temperature was held at $60^{\circ} \mathrm{C}$ for $1 \mathrm{~min}$, then raised to $210^{\circ} \mathrm{C}\left(10^{\circ} \mathrm{C} /\right.$ min), followed by a step to $230^{\circ} \mathrm{C}\left(5^{\circ} \mathrm{C} / \mathrm{min}\right)$ and reached $325^{\circ} \mathrm{C}\left(15^{\circ} \mathrm{C} / \mathrm{min}\right)$, and be hold at this temperature for $5 \mathrm{~min}$. The collision gas was nitrogen. The scan mode used was the MRM for biological samples. Peak detection and integration of the 133 analytes were performed using the Agilent Mass Hunter quantitative software (B.07.01).

(3) Targeted analysis of polyamines by ion pairing UHPLC coupled to a Triple Quadrupole (QQQ) mass spectrometer was performed on a RRLC 1260 system (Agilent Technologies, Waldbronn, Germany) coupled to a Triple Quadrupole 6410 (Agilent Technologies) equipped with an electrospray source operating in positive mode. The gas temperature was set to $350^{\circ} \mathrm{C}$ with a gas flow of $12 \mathrm{~L} / \mathrm{min}$. The capillary voltage was set to $3.5 \mathrm{kV}$. $10 \mu \mathrm{L}$ of sample were injected on a Column Kinetex C18 $(150 \mathrm{~mm} \times 2.1 \mathrm{~mm}$ particle size $2.6 \mu \mathrm{m})$ from Phenomenex, protected by a guard column $\mathrm{C} 18(5 \mathrm{~mm} \times 2.1 \mathrm{~mm})$ and heated at $40^{\circ} \mathrm{C}$ by a pelletier oven. The gradient mobile phase consisted of water with $0,1 \%$ of HFBA (A) and acetonitrile with $0,1 \%$ of HFBA (B) freshly made. The flow rate was set to $0.2 \mathrm{ml} /$ min, and gradient as follow: initial condition was $95 \%$ phase A and $5 \%$ phase B. Molecules were then eluted using a gradient from $5 \%$ to $40 \%$ phase B over $10 \mathrm{~min}$. The column was washed using $90 \%$ mobile phase B for $2.5 \mathrm{~min}$ and equilibrated using $5 \%$ mobile phase B for $4 \mathrm{~min}$. The autosampler was kept at $4{ }^{\circ} \mathrm{C}$. At the end of the batch of analysis, column was rinsed with $0.3 \mathrm{ml} / \mathrm{min}$ of MilliQ water (phase A) and acetonitrile (phase B) as follow: $10 \%$ phase B during $20 \mathrm{~min}$, to $90 \%$ phase B in $20 \mathrm{~min}$, and maintained during $20 \mathrm{~min}$ before shutdown. The collision gas was nitrogen. The scan mode used was the MRM for biological samples. Peak detection and integration of the 14 analytes were performed using the Agilent Mass Hunter quantitative software (B.07.01).

(4) Targeted analysis of bile acids by ion pairing UHPLC coupled to a Triple Quadrupole (QQQ) mass spectrometer was performed on a RRLC 1260 system (Agilent Technologies, Waldbronn, Germany) coupled to a Triple Quadrupole 6410 (Agilent Technologies) equipped with an electrospray source operating in positive mode. The gas temperature was set to $325^{\circ} \mathrm{C}$ with a gas flow of $12 \mathrm{~L} / \mathrm{min}$. The capillary voltage was set to $4.5 \mathrm{kV}$. $10 \mu \mathrm{L}$ of sample were injected on a Column Poroshell 120 EC-C8 $(100 \mathrm{~mm} \times 2.1 \mathrm{~mm}$ particle size $2.7 \mu \mathrm{m})$ from Agilent technologies, protected by a guard column XDB-C18 $(5 \mathrm{~mm} \times 2.1 \mathrm{~mm}$ particle size $1.8 \mu \mathrm{m})$ and heated at $40^{\circ} \mathrm{C}$ by a pelletier oven. The gradient mobile phase consisted of water with $0.2 \%$ of formic acid (A) and acetonitrile/isopropanol $(1 / 1$; $\mathrm{v} / \mathrm{v}$ ) (B) freshly made. The flow rate was set to $0.3 \mathrm{ml} / \mathrm{min}$, and gradient as follow: initial condition was $70 \%$ phase $\mathrm{A}$ and $30 \%$ phase $\mathrm{B}$, maintained during $1.5 \mathrm{~min}$. Molecules were then eluted using a gradient from $30 \%$ to $60 \%$ phase B over 9 min. The column was washed using $98 \%$ mobile phase B for 2 min and equilibrated using $30 \%$ mobile phase B for $2 \mathrm{~min}$. After each injection, the needle was washed twice with isopropanol and thrice with water. The autosampler was kept at $4{ }^{\circ} \mathrm{C}$. At the end of the batch of analysis, column was rinsed with $0.3 \mathrm{ml} / \mathrm{min}$ of MilliQ water (phase A) and acetonitrile (phase B) as follow: 10\% phase B during 20 min, to $90 \%$ phase B in $20 \mathrm{~min}$, and maintained during $20 \mathrm{~min}$ before shutdown. The collision gas was nitrogen. The scan mode used was the MRM for biological samples. Peak detection and integration of the 28 analytes were performed using the Agilent Mass Hunter quantitative software (B.07.01).

(5) The untargeted analysis of intracellular metabolites by UHPLC coupled to a Q-Exactive mass spectrometer using the Reversed-phase acetonitrile method was performed on a Dionex Ultimate 3000 UHPLC system (Thermo Scientific) coupled to a Q-Exactive (Thermo Scientific) equipped with an electrospray source operating in both positive and negative mode and full scan mode from 100 to $1200 \mathrm{~m} / z$. The Q-Exactive parameters were: sheath gas flow rate 50 au, auxiliary gas flow rate 10 au, spray voltage $4 \mathrm{kV}$, capillary temperature $300^{\circ} \mathrm{C}$, S-Lens RF level $55 \mathrm{~V} .10 \mu \mathrm{L}$ of sample were injected on a SB-Aq column $(100 \mathrm{~mm} \times 2.1 \mathrm{~mm}$ particle size $1.8 \mu \mathrm{m})$ from Agilent Technologies, protected by a guard column XDB-C18 $(5 \mathrm{~mm} \times 2.1$ $\mathrm{mm}$ particle size $1.8 \mu \mathrm{m}$ ) and heated at $40^{\circ} \mathrm{C}$ by a pelletier oven. The gradient mobile phase consisted of water with $0.2 \%$ of acetic acid (A) and acetonitrile (B) The flow rate was set to $0.5 \mathrm{ml} / \mathrm{min}$. Initial condition was $98 \%$ phase $\mathrm{A}$ and $2 \%$ phase B. Molecules were then eluted using a gradient from $2 \%$ to $95 \%$ phase B in 8 min. The column was washed using $95 \%$ mobile phase B for 3 min and equilibrated using $2 \%$ mobile phase B for $3 \mathrm{~min}$. The autosampler was kept at $4{ }^{\circ} \mathrm{C}$. At the end of the batch of analysis, column was rinsed with $0.4 \mathrm{ml} / \mathrm{min}$ of MilliQ water (phase A) and acetonitrile (phase B) as follow: 10\% phase B during 20 min, to $90 \%$ phase B in $20 \mathrm{~min}$, and maintained during $20 \mathrm{~min}$ before shutdown. Peak detection and integration were performed using the Thermo Xcalibur quantitative software (3.1.).

(6) Untargeted analysis of intracellular metabolites by UHPLC coupled to a QExactive mass spectrometer using the Reversed-phase methanol method was performed on a Dionex Ultimate 3000 UHPLC system (Thermo Scientific) coupled to a Q-Exactive (Thermo Scientific) equipped with an electrospray source operating in both positive and negative mode and full scan mode from 66 to $990 \mathrm{~m} / \mathrm{z}$. The QExactive parameters were: sheath gas flow rate 50 au, auxiliary gas flow rate $10 \mathrm{au}$, spray voltage $4 \mathrm{kV}$, capillary temperature $300^{\circ} \mathrm{C}$, S-Lens RF level $55 \mathrm{~V}$. Ten microlitres of sample were injected on an Eclipse Plus column $(100 \mathrm{~mm} \times 2.1 \mathrm{~mm}$ particle size $1.8 \mu \mathrm{m}$ ) from Agilent Technologies, protected by a guard column XDB-C18 $(5 \mathrm{~mm} \times 2.1 \mathrm{~mm}$ particle size $1.8 \mu \mathrm{m})$ and heated at $40^{\circ} \mathrm{C}$ by a pelletier oven. The gradient mobile phase consisted of water with $0.05 \%$ of formic acid (A) and methanol with $0.05 \%$ of formic acid (B). The flow rate was set to $0.35 \mathrm{ml} / \mathrm{min}$. Initial condition was $95 \%$ phase $\mathrm{A}$ and $5 \%$ phase B during $4 \mathrm{~min}$. Molecules were then eluted using a gradient from $5 \%$ to $60 \%$ phase B in $13 \mathrm{~min}$. The column was then washed using $90 \%$ mobile phase B for $2.5 \mathrm{~min}$ and equilibrated using $5 \%$ mobile phase B for $7.5 \mathrm{~min}$. The autosampler was kept at $4{ }^{\circ} \mathrm{C}$. At the end of the batch of analysis, column was rinsed with $0.4 \mathrm{ml} / \mathrm{min}$ of MilliQ water (phase A) and acetonitrile (phase B) as follow: $10 \%$ phase B during $20 \mathrm{~min}$, to $90 \%$ phase B in $20 \mathrm{~min}$, and maintained during $20 \mathrm{~min}$ before shutdown. Peak detection and integration were performed using the Thermo Xcalibur quantitative software (3.1.)

(7) Untargeted analysis of intracellular metabolites by UHPLC coupled to a QExactive mass spectrometer using the HILIC method was performed on a Dionex Ultimate 3000 UHPLC system (Thermo Scientific) coupled to a Q-Exactive (Thermo Scientific) equipped with an electrospray source operating in both positive and negative mode and full scan mode from 66 to $990 \mathrm{~m} / \mathrm{z}$. The Q-Exactive parameters were: sheath gas flow rate $50 \mathrm{au}$, auxiliary gas flow rate $10 \mathrm{au}$, spray voltage $4 \mathrm{kV}$, capillary temperature $300^{\circ} \mathrm{C}$, S-Lens RF level $55 \mathrm{~V}$. The sample were diluted with a final volume of $50 \%$ acetonitrile, then $5 \mu \mathrm{l}$ were injected on a SeQuant ZIC-pHILIC $(150 \mathrm{~mm} \times 2.1 \mathrm{~mm}$ particle size $5 \mu \mathrm{m})$ from Merck Millipore, protected by a guard column SeQuant ZIC-pHILIC $(20 \mathrm{~mm} \times 2.1 \mathrm{~mm}$ particle size $5 \mu \mathrm{m}$ ) and heated at $40{ }^{\circ} \mathrm{C}$ by a pelletier oven. The gradient mobile phase consisted of water with $20 \mathrm{mM}$ of ammonium carbonate (A) and acetonitrile (B). The flow rate was set to $0.3 \mathrm{ml} / \mathrm{min}$. Initial condition was $5 \%$ phase A and $95 \%$ phase B during $1 \mathrm{~min}$. Molecules were then eluted using a gradient as follow: $92 \%$ in $2 \mathrm{~min}, 86.5 \%$ in $0.5 \mathrm{~min}, 65 \%$ in $17.5 \mathrm{~min}$ of mobile phase B. The column was washed using $10 \%$ mobile phase $\mathrm{B}$ for $4.5 \mathrm{~min}$ and then equilibrated for the next analysis using $95 \%$ mobile phase B for $9 \mathrm{~min}$. The autosampler was kept at $4{ }^{\circ} \mathrm{C}$. At the end of the batch of analysis, column was rinsed with $0.3 \mathrm{ml} / \mathrm{min}$ of water $5 \mathrm{mM}$ acetate ammonium (phase A) and acetonitrile (phase B) as follow: $50 \%$ phase B during $21 \mathrm{~min}$, to $80 \%$ phase B in $21 \mathrm{~min}$, and maintained during $18 \mathrm{~min}$ before shutdown. To remove residual salts from tubing, additional rinse of the autosampler (water/acetonitrile; $9 / 1 ; \mathrm{v} / \mathrm{v}$ ) was added during $15 \mathrm{~min}$, with the column off-line. Peak detection and integration were performed using the Thermo Xcalibur quantitative software (3.1.). The complete data set is available in Supplementary Data Files 3 and 4.

Metabolite clustering. Hierarchical clustering was performed using the MetaboAnalyst 3.0 suite $^{71}$ with the $\log 2$-normalised data as input using following parameters (unless stated otherwise): missing value estimation: skipped; data normalisation: none; data transformation: none; data scaling: none; distance measure: Pearson; clustering algorithm: Ward; default colour contrast; top 50 metabolites ranked by $t$-tests; data standardisation: autoscale features. Combined pathway topology and enrichment analysis was performed using the pathway analysis tool of MetaboAnalyst 3.0 with following parameters: missing value estimation: skipped; data normalization: none; data transformation: none; data scaling: none; pathway enrichment analysis: global test; pathway topology analysis: relativebetweenness centrality; reference: all compounds of the selected pathways. Pathways with impact " 0 " were omitted. As hexose-phosphate cannot be clearly 
assigned to a specific metabolite, it was excluded for yeast pathway topology analysis. The complete pathway topology analyses are available in Supplementary Data File 1 (yeast), Supplementary Data File 3 (mouse heart) and Supplementary Data File 4 (mouse liver).

Measurement of infarct size. Three hours after LAD ligation, the animals were anesthetised and intubated, and the chest was opened. To demarcate the ischaemic area-at-risk (AAR), Alcian blue dye (1\%) was perfused into the aorta and coronary arteries. Hearts were excised, and LVs were sliced into 1-mm-thick cross sections. The heart sections were then incubated with a $1 \%$ triphenyltetrazolium chloride solution at $37^{\circ} \mathrm{C}$ for $15 \mathrm{~min}$. The infarct area (white), the AAR (not blue), and the total LV area from both sides of each section were measured using ImageJ software and the values obtained were averaged. The percentage of area of infarction and AAR of each section were multiplied by the weight of the section and then totaled from all sections. AAR/LV and infarct area/AAR were expressed as percentages.

Ethanol intoxication and determination of ALT activity. Mice were fasted for $6 \mathrm{~h}$ and then they were administered a $33 \%(\mathrm{vol} / \mathrm{vol})$ ethanol solution at a total cumulative dosage of $4.5-\mathrm{g} / \mathrm{kg}$ by four equally divided gavages in 20 -minute intervals, as described in Ding et al. ${ }^{72}$. Control mice received the same volume of water. Vehicle (DMSO) or DMC were intraperitoneally injected to mice $30 \mathrm{~min}$ before ethanol administration. Sub-mandibular blood collection occurred $16 \mathrm{~h}$ after ethanol binge. Hepatic damage was quantified as serum ALT activity (Alanine Aminotransferase activity) by means of a specific kit (Alanine Transaminase Activity Assay Kit, Abcam [ab105134]). ALT activity was calculated as [ $\left.\mathrm{mU}^{*} \mathrm{mg}^{-1}\right]$ of pyruvate produced in the reaction.

Extractions and measurements of DMC. For DMC extraction of plant samples, $10 \mathrm{mg}$ of plant powder were placed into $1 \mathrm{ml}$ of ethylacetate and the samples extracted at room temperature for an hour in an ultrasonic bath followed by another hour on a shaker at $145 \mathrm{rpm}$ (motor speed). Afterwards, the samples were centrifuged, the supernatant evaporated using nitrogen flow at room temperature and the resulting residue reconstituted in $100 \mu \mathrm{l} 60 \%$ acetonitrile. For DMC extraction of mice plasma, $40 \mu \mathrm{l}$ of plasma were precipitated with $150 \mu \mathrm{l}$ of acetonitrile, vortexed, and centrifuged. The supernatant was evaporated at room temperature under nitrogen flow. The residue was dissolved in $50 \mu \mathrm{l}$ of $60 \%$ acetonitrile.

All obtained samples were analysed using LC/MS. Experiments were carried out on an Ultimate 3000 System (Dionex, LCPackings, Vienna, Austria) coupled to a LTQ Orbitrap (ThermoFinnigan, Vienna, Austria), using an ESI ion source in positive mode. The system was controlled by Xcalibur Software 1.4. Chromatography was carried out on a Gemini C18, 20 x 5 mm $3 \mu \mathrm{m}$ (Phenomenex, Torrance, USA) column using $0.1 \%$ formic acid in water (A) and $0.1 \%$ formic acid in acetonitrile (B) as mobile phase. The separation was performed under gradient elution conditions: $60 \%$ B up to $90 \%$ B in 8.5 min, keeping $90 \%$ B constant for 3 min and re-equilibrating for $4 \mathrm{~min}$. The flow rate was $200 \mu \mathrm{l} / \mathrm{min}$ at $30^{\circ} \mathrm{C}$, injection volume $20 \mu \mathrm{l}$. For the mass spectrometry measurements 2 scan events were monitored simultaneously: scan mode in the FTMS and production ion scan of 269 (CID 35) in the LTQ ion trap.

Immunoblotting. Preparation of yeast cell extracts for the EGFP-Atg8 autophagy assay and expression levels of HA-tagged Atg proteins were performed as described $^{17}$. Therefore, three $\mathrm{OD}_{600}$ units of cells $\left(\sim 1 \times 10^{8}\right.$ cells $)$ were harvested by centrifugation at $10,000 \times g$ for $1 \mathrm{~min}$, washed once with $500 \mu \mathrm{l} \mathrm{H}_{2} \mathrm{O}$ and resuspended in $300 \mu \mathrm{l}$ lysis buffer (1.85 M NaOH, 7.5\% 2-mercaptoethanol) and incubated for $10 \mathrm{~min}$ on ice. Then, $300 \mu \mathrm{l} 55 \%$ trichloroacetic acid were added, samples were mixed briefly and incubated for another $10 \mathrm{~min}$ on ice. The precipitate was pelleted by centrifugation at $10,000 \times g$ at $4{ }^{\circ} \mathrm{C}$ for $10 \mathrm{~min}$ and the supernatant was removed (followed by a short spin to remove residual supernatant). The pellet was resuspended in $150 \mu \mathrm{l}$ sample buffer $(200 \mathrm{mM}$ Tris/ $\mathrm{HCl} \mathrm{pH} 6.8,2 \%$ SDS, $10 \%$ glycerol, $120 \mathrm{mM}$ DTT, $0.004 \%$ bromophenol blue), placed in an ultrasound bath for $5 \mathrm{~min}$ and then boiled for $5 \mathrm{~min}$ at $95^{\circ} \mathrm{C}$. Before gel electrophoresis, samples were centrifuged at $10,000 \times g$ for $1 \mathrm{~min}$.

Immunoblotting was performed using standard protocols. Blots were probed with murine monoclonal antibodies against GFP (Roche, [\#1814460], 1:5,000 in TBS $+1 \%$ dry milk) or HA (Sigma-Aldrich, [H-9658], 1:10,000 in TBS $+1 \%$ dry milk) and the respective peroxidase-conjugated affinity-purified secondary antibody (Sigma, [\#F-9137], 1:10,000 in TBS + 1\% dry milk).

For human cell culture experiments, proteins were extracted from cells by means of Radio Immunoprecipitation Assay (RIPA) buffer. Twenty-five micrograms of proteins were separated on $4-12 \%$ bis-tris acrylamide (Thermo Fisher Scientific Inc.) and electrotransferred to Immun-Blot ${ }^{\circledR}$ PVDF Membrane (Biorad) Membranes were then sliced horizontally in different parts according to the molecular weight of the protein of interest to allow simultaneous detection of different antigens within the same experiment. Unspecific binding sites were saturated by incubating membranes for $1 \mathrm{~h}$ in $0.05 \%$ Tween 20 (v:v in TBS) supplemented with $5 \%$ non-fat powdered milk (w:v in TBS), followed by an overnight incubation with primary antibodies specific for LC3B (Cell Signalling Technology, [\#2775], rabbit, 1:1,000) and SQSTM1/p62 (Abnova, clone 2C11,
[\#H00008878-M01], mouse, 1:10,000). Development was performed with appropriate horseradish peroxidase (HRP)-labelled secondary antibodies (RabbitHRP, Thermo Scientific, [\#31460], 1:5,000; Mouse-HRP, Thermo Scientific, [\#31430], 1:5,000) plus the Super Signal West Pico chemiluminescent substrate (Thermo Fisher Scientific Inc.). An anti-glyceraldehyde-3-phosphate dehydrogenase antibody was used to control equal loading of lanes (for yeast experiments: the antibody was a gift from Dr. Guenther Daum, rabbit, 1:40,000; for human cell and mouse experiments: Cell Signalling Technology, clone D16H11, [\#5174], rabbit, 1:10,000). For knockdown efficiency tests of GATA siRNA and controls (Supplementary Figure 13), the following antibodies were used: Thermo Fisher Scientific \#PA1099X, rabbit, 1:500 (GATA1), Thermo Fisher Scientific [\#710242], rabbit, 1:100 (GATA2), Cell Signalling Technology [\#5852], rabbit, 1:1000 (GATA3), Cell Signalling Technology [\#36966], rabbit, 1:1000 (GATA4), Thermo Fisher Scientific [\#PA547262], goat, 1:200 (GATA5), Cell Signalling Technology [\#5851], rabbit, 1:1000 (GATA6), Abcam [\#ab48820], rabbit, 1:2000 (TRPS1), Cell Signalling Technology [\#12994], rabbit, 1:1000 (Atg5). Full scans of all immunoblots are depicted in Supplementary Fig. 17.

Statistical analyses. The number of independent biological replicates $(n)$ is indicated in the figure legends of the corresponding graphs. If not stated otherwise, independence is defined for yeast experiments as different clones or transformants, aged separately; for cell culture as separately treated cultures; for C. elegans, Drosophila and mouse experiments as different animals. Sample size was preestimated based on the effect size observed in initial yeast experiments. There was no blinding in the experiments. Where normality criteria were met (calculated by Kolmogorow-Smirnow and Bartlett's tests), $P$-values were calculated using Student's $t$-test (two-sided, unpaired) or an analysis of variance (ANOVA) approach (for more than two groups), followed by a Bonferroni post hoc test. Nonparametric data was analysed using a Kruskal-Wallis test with Dunn's multiple comparison correction. Where necessary, data was log-transformed to achieve normality and homogenous variances and significance assessed for the transformed data. For data with two variables (e.g. treatment and strain) a two-way ANOVA was performed, followed by testing simple main effects (Bonferroni-corrected multiple comparisons of different levels of each factor in case of significant main factor or interaction). Significance of yeast ageing survival data (factors treatment and time) was tested using repeated measures ANOVA (two-way mixed ANOVA) with time as the within-subjects factor. For survival analyses, log-rank (Mantel-Cox) tests were performed between DMC-treated and untreated conditions and the significance threshold was adapted according to the number of pairwise comparisons. If not stated otherwise, asterisks indicate significance: ${ }^{* *} P<$ $0.001,{ }^{*} P<0.01,{ }^{\star} P<0.05$. Statistics were performed using Origin Pro 2008 (OriginLab).

Reporting summary. Further information on experimental design is available in the Nature Research Reporting Summary linked to this article.

\section{Data availability}

The raw mass spectrometry proteomics data to the ProteomeXchange Consortium via the PRIDE partner repository; the project accession number is PXD012108. The metabolomics data are available at Mendeley Data, [https://doi.org/10.17632/83xw8jtgh2.1] X. In addition, all raw and processed LC-MS/MS metabolomics and proteomics data are available as Supplementary Data files 1-4. The source data underlying Fig. 1 (b-f, j), 2 (b, $\mathrm{d}-\mathrm{e}, \mathrm{g}, \mathrm{i}), 3$ (a, c), 4 (a), 5 (a-b, d-g), 6 (a-b, d) and 7 (c-d) as well as those underlying Supplementary Figures 1 (a-e), 2 (a-d, e-f), 4 (a-b), 5 (d-e), 6 (b, d, f, h-k, m-o), 7 (a-b, h) 8 (a-e), 9 (a-e), $10(\mathrm{a}-\mathrm{g}), 11$ (a-e, g), 14 (a) and 16 (a) are provided as a Source Data file. All other data supporting the findings of this study are available from the corresponding authors on reasonable request. A reporting summary for this Article is available as a Supplementary Information file.

Received: 30 October 2018 Accepted: 11 January 2019 Published online: 19 February 2019

\section{References}

1. Niccoli, T. \& Partridge, L. Ageing as a risk factor for disease. Curr. Biol. 22, R741-R752 (2012).

2. de Cabo, R., Carmona-Gutierrez, D., Bernier, M., Hall, M. N. \& Madeo, F. The search for antiaging interventions: from elixirs to fasting regimens. Cell 157, 1515-1526 (2014).

3. Bonkowski, M. S. \& Sinclair, D. A. Slowing ageing by design: the rise of NAD +and sirtuin-activating compounds. Nat. Rev. Mol. Cell Biol. 17, 679-690 (2016).

4. Uysal, U. et al. Consumption of polyphenol plants may slow aging and associated diseases. Curr. Pharm. Des. 19, 6094-6111 (2013).

5. Baur, J. A. et al. Resveratrol improves health and survival of mice on a highcalorie diet. Nature 444, 337-342 (2006). 
6. Bisht, K., Wagner, K.-H. \& Bulmer, A. C. Curcumin, resveratrol and flavonoids as anti-inflammatory, cyto- and DNA-protective dietary compounds. Toxicology 278, 88-100 (2010).

7. Singh, P., Anand, A. \& Kumar, V. Recent developments in biological activities of chalcones: a mini review. Eur. J. Med. Chem. 85, 758-777 (2014).

8. Yin, Z., Pascual, Clarence \& Klionsky, DanielJ. Autophagy: machinery and regulation. Microb. Cell 3, 457-465 (2016)

9. Jiang, P. \& Mizushima, N. Autophagy and human diseases. Cell Res. 24, 69-79 (2014).

10. Nixon, R. A. The role of autophagy in neurodegenerative disease. Nat. Med. 19, 983-997 (2013).

11. Fabrizio, P., Pozza, F., Pletcher, S. D., Gendron, C. M. \& Longo, V. D. Regulation of longevity and stress resistance by Sch9 in yeast. Science 292, 288-290 (2001).

12. Fontana, L., Partridge, L. \& Longo, V. D. Extending healthy life span--from yeast to humans. Science 328, 321-326 (2010).

13. Carmona-Gutierrez, D. et al. Guidelines and recommendations on yeast cell death nomenclature. Microb. Cell 5, 4-31 (2018).

14. Pallauf, K. \& Rimbach, G. Autophagy, polyphenols and healthy ageing. Ageing Res. Rev. 12, 237-252 (2013)

15. Klionsky, D. J. et al. Guidelines for the use and interpretation of assays for monitoring autophagy (3rd edition). Autophagy 12, 1-222 (2016).

16. Noda, T. \& Klionsky, D. J. The quantitative Pho8Delta60 assay of nonspecific autophagy. Methods Enzymol. 451, 33-42 (2008).

17. Eisenberg, T. et al. Nucleocytosolic depletion of the energy metabolite acetylcoenzyme A stimulates autophagy and prolongs lifespan. Cell. Metab. 19, 431-444 (2014).

18. Matsui, Y. et al. Distinct roles of autophagy in the heart during ischemia and reperfusion: roles of AMP-activated protein kinase and Beclin 1 in mediating autophagy. Circ. Res. 100, 914-922 (2007).

19. Shirakabe, A., Ikeda, Y., Sciarretta, S., Zablocki, D. K. \& Sadoshima, J. Aging and Autophagy in the Heart. Circ. Res. 118, 1563-1576 (2016).

20. Lakatta, E. G. \& Levy, D. Arterial and cardiac aging: major shareholders in cardiovascular disease enterprises: Part I: aging arteries: a 'set up' for vascular disease. Circulation 107, 139-146 (2003)

21. Lőw, P. et al. Impaired proteasomal degradation enhances autophagy via hypoxia signaling in Drosophila. BMC Cell. Biol. 14, 29 (2013).

22. Deegan, S., Saveljeva, S., Gorman, A. M. \& Samali, A. Stress-induced selfcannibalism: on the regulation of autophagy by endoplasmic reticulum stress. Cell. Mol. Life Sci. 70, 2425-2441 (2013).

23. Cebollero, E. \& Reggiori, F. Regulation of autophagy in yeast Saccharomyces cerevisiae. Biochim. Biophys. Acta 1793, 1413-1421 (2009).

24. Reggiori, F. \& Klionsky, D. J. Autophagic processes in yeast: mechanism, machinery and regulation. Genetics 194, 341-361 (2013).

25. Tate, J. J., Georis, I., Dubois, E. \& Cooper, T. G. Distinct phosphatase requirements and GATA factor responses to nitrogen catabolite repression and Rapamycin treatment in Saccharomyces cerevisiae. J. Biol. Chem. 285, 17880-17895 (2010).

26. Ljungdahl, P. O. \& Daignan-Fornier, B. Regulation of amino acid, nucleotide, and phosphate metabolism in Saccharomyces cerevisiae. Genetics 190, 885-929 (2012).

27. Mirzaei, H., Suarez, J. A. \& Longo, V. D. Protein and amino acid restriction, aging and disease: from yeast to humans. Trends Endocrinol. Metab. TEM 25, 558-566 (2014).

28. Grandison, R. C., Piper, M. D. W. \& Partridge, L. Amino-acid imbalance explains extension of lifespan by dietary restriction in Drosophila. Nature 462, 1061-1064 (2009).

29. Vlahakis, A., Graef, M., Nunnari, J. \& Powers, T. TOR complex 2-Ypk1 signaling is an essential positive regulator of the general amino acid control response and autophagy. Proc. Natl Acad. Sci. USA 111, 10586-10591 (2014).

30. Mülleder, M. et al. Functional metabolomics describes the yeast biosynthetic regulome. Cell 167, 553-565 (2016). e12.

31. Devasahayam, G., Ritz, D., Helliwell, S. B., Burke, D. J. \& Sturgill, T. W. Pmr1, a Golgi Ca2+/Mn2+-ATPase, is a regulator of the target of rapamycin (TOR) signaling pathway in yeast. Proc. Natl Acad. Sci. USA 103, 17840-17845 (2006).

32. Cooper, T. G. Transmitting the signal of excess nitrogen in Saccharomyces cerevisiae from the Tor proteins to the GATA factors: connecting the dots. FEMS Microbiol. Rev. 26, 223-238 (2002).

33. Johnson, S. C., Rabinovitch, P. S. \& Kaeberlein, M. mTOR is a key modulator of ageing and age-related disease. Nature 493, 338-345 (2013).

34. Jiang, Y. \& Broach, J. R. Tor proteins and protein phosphatase $2 \mathrm{~A}$ reciprocally regulate Tap42 in controlling cell growth in yeast. EMBO J. 18, 2782-2792 (1999).

35. Tate, J. J., Georis, I., Feller, A., Dubois, E. \& Cooper, T. G. Rapamycin-induced Gln3 dephosphorylation is insufficient for nuclear localization. J. Biol. Chem. 284, 2522-2534 (2009).
36. Liu, M., Wilairat, P. \& Go, M.-L. Antimalarial alkoxylated and hydroxylated chalones: structure-activity relationship analysis. J. Med. Chem. 44, 4443-4452 (2001)

37. Ducki, S. et al. Combretastatin-like chalcones as inhibitors of microtubule polymerization. Part 1: Synthesis and biological evaluation of antivascular activity. Bioorg. Med. Chem. 17, 7698-7710 (2009).

38. Vaidya, S. S., Vinaya, H. \& Mahajan, S. S. Microwave-assisted synthesis, pharmacological evaluation, and QSAR studies of 1,3-diaryl-2-propen-1-ones. Med. Chem. Res. 21, 4311-4323 (2012).

39. Rice-Evans, C. A. \& Miller, N. J. Antioxidant activities of flavonoids as bioactive components of food. Biochem. Soc. Trans. 24, 790-795 (1996).

40. Lu, M.-F., Xiao, Z.-T. \& Zhang, H.-Y. Where do health benefits of flavonoids come from? Insights from flavonoid targets and their evolutionary history. Biochem. Biophys. Res. Commun. 434, 701-704 (2013).

41. Powers, R. W., Kaeberlein, M., Caldwell, S. D., Kennedy, B. K. \& Fields, S. Extension of chronological life span in yeast by decreased TOR pathway signaling. Genes Dev. 20, 174-184 (2006).

42. Chan, T. F., Bertram, P. G., Ai, W. \& Zheng, X. F. Regulation of APG14 expression by the GATA-type transcription factor Gln3p. J. Biol. Chem. 276, 6463-6467 (2001).

43. Mizushima, N., Yoshimori, T. \& Levine, B. Methods in mammalian autophagy research. Cell 140, 313-326 (2010).

44. Bernard, A., Jin, M., Xu, Z. \& Klionsky, D. J. A large-scale analysis of autophagy-related gene expression identifies new regulators of autophagy. Autophagy 11, 2114-2122 (2015).

45. Rai, R., Tate, J. J. \& Cooper, T. G. Multiple targets on the Gln3 transcription activator are cumulatively required for control of its cytoplasmic sequestration. G3 Bethesda Md 6, 1391-1408 (2016).

46. Tate, J. J., Buford, D., Rai, R. \& Cooper, T. G. General amino acid control and 14-3-3 proteins Bmh1/2 are required for nitrogen catabolite repressionsensitive regulation of Gln3 and Gat1 localization. Genetics 205, 633-655 (2017).

47. Tate, J. J., Rai, R. \& Cooper, T. G. More than one way in: three Gln3 sequences required to relieve negative Ure2 regulation and support nuclear Gln3 import in Saccharomyces cerevisiae. Genetics 208, 207-227 (2018).

48. Zhang, P., Judy, M., Lee, S.-J. \& Kenyon, C. Direct and indirect gene regulation by a life-extending FOXO protein in C. elegans: roles for GATA factors and lipid gene regulators. Cell. Metab. 17, 85-100 (2013).

49. Bresnick, E. H., Lee, H.-Y., Fujiwara, T., Johnson, K. D. \& Keles, S. GATA switches as developmental drivers. J. Biol. Chem. 285, 31087-31093 (2010).

50. Kang, Y.-A. et al. Autophagy driven by a master regulator of hematopoiesis. Mol. Cell. Biol. 32, 226-239 (2012).

51. Kobayashi, S. et al. Transcription factor GATA4 inhibits doxorubicin-induced autophagy and cardiomyocyte death. J. Biol. Chem. 285, 793-804 (2010).

52. Eisenberg, T. et al. Induction of autophagy by spermidine promotes longevity. Nat. Cell Biol. 11, 1305-1314 (2009).

53. Janke, C. et al. A versatile toolbox for PCR-based tagging of yeast genes: new fluorescent proteins, more markers and promoter substitution cassettes. Yeast Chichester Engl. 21, 947-962 (2004).

54. Rai, R. et al. Nuclear Gln3 import is regulated by nitrogen catabolite repression whereas export is specifically regulated by glutamine. Genetics 201, 989-1016 (2015).

55. Herker, E. et al. Chronological aging leads to apoptosis in yeast. J. Cell. Biol 164, 501-507 (2004).

56. Burtner, C. R., Murakami, C. J., Kennedy, B. K. \& Kaeberlein, M. A molecular mechanism of chronological aging in yeast. Cell Cycle Georget. Tex. 8, 1256-1270 (2009).

57. Jung, P. P., Christian, N., Kay, D. P., Skupin, A. \& Linster, C. L. Protocols and programs for high-throughput growth and aging phenotyping in yeast. PLoS ONE 10, e0119807 (2015)

58. Gueldener, U., Heinisch, J., Koehler, G. J., Voss, D. \& Hegemann, J. H. A second set of loxP marker cassettes for Cre-mediated multiple gene knockouts in budding yeast. Nucleic Acids Res. 30, e23 (2002).

59. Carmona-Gutiérrez, D. et al. The propeptide of yeast cathepsin D inhibits programmed necrosis. Cell Death Dis. 2, e161 (2011).

60. González, A. et al. TORC1 promotes phosphorylation of ribosomal protein S6 via the AGC kinase Ypk3 in Saccharomyces cerevisiae. PLoS ONE 10, e0120250 (2015).

61. Buescher, J. M., Moco, S., Sauer, U. \& Zamboni, N. Ultrahigh performance liquid chromatography-tandem mass spectrometry method for fast and robust quantification of anionic and aromatic metabolites. Anal. Chem. 82, 4403-4412 (2010)

62. Fröhlich, E. E. et al. Cognitive impairment by antibiotic-induced gut dysbiosis: Analysis of gut microbiota-brain communication. Brain Behav. Immun. 56, 140-155 (2016).

63. Rappsilber, J., Mann, M. \& Ishihama, Y. Protocol for micro-purification, enrichment, pre-fractionation and storage of peptides for proteomics using StageTips. Nat. Protoc. 2, 1896-1906 (2007). 
64. Cox, J. \& Mann, M. MaxQuant enables high peptide identification rates, individualized p.p.b.-range mass accuracies and proteome-wide protein quantification. Nat. Biotechnol. 26, 1367-1372 (2008).

65. Vizcaíno, J. A. et al. 2016 update of the PRIDE database and its related tools. Nucleic Acids Res. 44, D447-D456 (2016).

66. Stiernagle, T. Maintenance of C. elegans. WormBook Online Rev. C Elegans Biol. 1-11 (2006). https://doi.org/10.1895/wormbook.1.101.1

67. Kamath, R. S. \& Ahringer, J. Genome-wide RNAi screening in Caenorhabditis elegans. Methods San. Diego Calif. 30, 313-321 (2003).

68. Rea, S. L., Ventura, N. \& Johnson, T. E. Relationship between mitochondrial electron transport chain dysfunction, development, and life extension in Caenorhabditis elegans. PLoS Biol. 5, e259 (2007).

69. Juhász, G., Érdi, B., Sass, M. \& Neufeld, T. P. Atg7-dependent autophagy promotes neuronal health, stress tolerance, and longevity but is dispensable for metamorphosis in Drosophila. Genes Dev. 21, 3061-3066 (2007).

70. Leontieva, O. V. \& Blagosklonny, M. V. Yeast-like chronological senescence in mammalian cells: phenomenon, mechanism and pharmacological suppression. Aging 3, 1078-1091 (2011).

71. Xia, J. \& Wishart, D. S. Using MetaboAnalyst 3.0 for Comprehensive Metabolomics Data Analysis. Curr. Protoc. Bioinforma. 55, 14.10.1-14.10.91 (2016).

72. Ding, W.-X. et al. Autophagy reduces acute ethanol-induced hepatotoxicity and steatosis in mice. Gastroenterology 139, 1740-1752 (2010).

\section{Acknowledgements}

F.M. and D.C-G. are grateful to the Austrian Science Fund FWF (SFB-LIPOTOX F3007\&F3012, W1226, P29203, P29262), in particular for the project P27893 ("Proautophagic polyphenols and polyamines for longevity") and the Austrian Federal Ministry of Education, Science and Research and the University of Graz for grants "Unkonventionelle Forschung" and "flysleep" (BMWFW-80.109/0001-WF/V/3b/2015) J.T. is funded by the FWF (W 1226, DK Metabolic and Cardiovascular Disease) at the University of Graz. K.K. is a fellow of the Doctoral College "Metabolic and Cardiovascular Disease" (FWF W1226) and was funded by the University of Graz. We acknowledge support from NAWI Graz and the BioTechMed-Graz flagship project "EPIAge". G. $\mathrm{K}$. is supported by the Ligue contre le Cancer (équipe labelisée); Agence National de la Recherche (ANR) - Projets blancs; ANR under the frame of E-Rare-2, the ERA-Net for Research on Rare Diseases; Association pour la recherche sur le cancer (ARC); Cancéropôle Ile-de-France; Institut National du Cancer (INCa); Inserm (HTE); Institut Universitaire de France; Fondation pour la Recherche Médicale (FRM); the European Commission (ArtForce); the European Research Council (ERC); Fondation Carrefour; the LeDucq Foundation; the LabEx Immuno-Oncology; the RHU Torino Lumière, the Seerave Foundation, the SIRIC Stratified Oncology Cell DNA Repair and Tumour Immune Elimination (SOCRATE); the SIRIC Cancer Research and Personalised Medicine (CARPEM); and the Paris Alliance of Cancer Research Institutes (PACRI). N.V. would like to thank the Deutsche Forschungsgemeinschaft (DFG) and the Bundesministerium für Bildung und Forschung (BMBF) for funding. We thank Dr. Stefan Benke and Lydia Opriessnig for technical assistance and Dr. Sabrina Büttner as well as Dr. Ulrich Stelzl and Katharina Radakovics for advice and discussions. We thank Dr. Tarek Moustafa for providing the rps6 mutant strain and Dr. Carlos Lopez-Otin (Oviedo University, Spain) for kindly providing Atg $4 \mathrm{~b}^{-1-}$ mice. We further thank Dr. Bruno André (Université Libre de Bruxelles, Belgium) for providing the PMEP2-LacZ fusion plasmid. We thank Dr. Guenther Daum (Technical University of Graz, Austria) for providing the GAPDH antibody for yeast western blot analyses. We also thank Dr. Gabor Juhasz (Eötvös Loránd University, Hungary) for providing the anti-Ref(2)P antibody for corresponding measurements in fly brains.

\section{Author contributions}

D.C.-G., A.Z., G.K. and F.M. designed the research; D.C.-G., A.Z., K.K., S.Sc., S.J.H., M.A B., T.P., J.T., C.D. and C.R. performed the yeast experiments; F.P., G.C., F.C. and V.S. performed the mouse and cell culture experiments; S.Ma., A.S., and N.V. designed and performed the nematode experiments; A.Z., K.K., S.J.H., C.S., C.B.B., S.Me., H.W. and S.J.S. designed and performed the Drosophila experiments; G.T., R.R. and C.M. performed the metabolomics from yeast and measurements from Ashitaba extracts; Z.H. and J.D. performed the proteomics; J.N. and J.S. performed the ischemia experiments; F.P., S.D., N.B., F.A., O.K. and D.P.E. performed the mouse metabolomics. T.E., F.S., M.A., S.Se. and T.R.P. helped with experimental design; D.C.-G. and A.Z. analysed most of the data and D.C.-G., A.Z., G.K. and F.M. wrote the manuscript.

\section{Additional information}

Supplementary Information accompanies this paper at https://doi.org/10.1038/s41467 019-08555-w.

Competing interests: D.C-G., G.K., O.K. and F.M. are the scientific co-founders of Samsara Therapeutics. The remaining authors declare no competing interests.

Reprints and permission information is available online at http://npg.nature.com/ reprintsandpermissions/

Journal peer review information: Nature Communications thanks the anonymous reviewers for their contribution to the peer review of this work. Peer reviewer reports are available.

Publisher's note: Springer Nature remains neutral with regard to jurisdictional claims in published maps and institutional affiliations.

\begin{abstract}
Open Access This article is licensed under a Creative Commons Attribution 4.0 International License, which permits use, sharing, adaptation, distribution and reproduction in any medium or format, as long as you give appropriate credit to the original author(s) and the source, provide a link to the Creative Commons license, and indicate if changes were made. The images or other third party material in this article are included in the article's Creative Commons license, unless indicated otherwise in a credit line to the material. If material is not included in the article's Creative Commons license and your intended use is not permitted by statutory regulation or exceeds the permitted use, you will need to obtain permission directly from the copyright holder. To view a copy of this license, visit http://creativecommons.org/ licenses/by/4.0/.
\end{abstract}

(C) The Author(s) 2019

Didac Carmona-Gutierrez ${ }^{1}$, Andreas Zimmermann (1) ${ }^{1,2}$, Katharina Kainz' ${ }^{1}$, Federico Pietrocola ${ }^{3,4,5,6}$, Guo Chen ${ }^{3,4,5,6}$, Silvia Maglioni ${ }^{7}$, Alfonso Schiavi ${ }^{7}$, Jihoon $\mathrm{Nah}^{8}$, Sara Mertel ${ }^{9}$, Christine B. Beuschel ${ }^{9}$, Francesca Castoldi ${ }^{3,4,5,6,10}$, Valentina Sica ${ }^{3,4,5,6}$, Gert Trausinger ${ }^{11}$, Reingard Raml ${ }^{17}$, Cornelia Sommer ${ }^{1}$, Sabrina Schroeder', Sebastian J. Hofer ${ }^{1}{ }^{1}$, Maria A. Bauer', Tobias Pendl', Jelena Tadic', Christopher Dammbrueck', Zehan Hu'2, Christoph Ruckenstuhl', Tobias Eisenberg (1) ', Sylvere Durand ${ }^{3,4}$, Noélie Bossut ${ }^{3,4}$, Fanny Aprahamian ${ }^{3,4}$, Mahmoud Abdellatif ${ }^{12}$, Simon Sedej ${ }^{12,13}$, David P. Enot ${ }^{3,4}$, Heimo Wolinski ${ }^{1}$, Jörn Dengjel $\oplus^{14}$, Oliver Kepp $\circledast^{3,4,5,6}$, Christoph Magnes ${ }^{11}$, Frank Sinner ${ }^{2,11}$, Thomas R. Pieber, ${ }^{2,11}$, Junichi Sadoshima ${ }^{8}$, Natascia Ventura $\mathbb{1}^{7,15}$, Stephan J. Sigrist ${ }^{9,16}$, Guido Kroemer $3,4,5,6,17,18$ \& Frank Madeo $0^{1,13}$

${ }^{1}$ Institute of Molecular Biosciences, NAWI Graz, University of Graz, Graz 8010, Austria. ${ }^{2}$ Division of Endocrinology and Diabetology, Department of Internal Medicine, Medical University of Graz, Graz 8036, Austria. ${ }^{3}$ Equipe 11 labellisée Ligue contre le Cancer, Centre de Recherche des Cordeliers, INSERM U 1138, Paris, France. ${ }^{4}$ Metabolomics and Cell Biology Platforms, Gustave Roussy Comprehensive Cancer Center, Villejuif, France. ${ }^{5}$ Université Paris Descartes, Sorbonne Paris Cité, Paris, France. ${ }^{6}$ Université Pierre et Marie Curie, Paris, France. ${ }^{7}$ IUF - Leibniz Research Institute for 
Environmental Medicine, Düsseldorf 40225, Germany. ${ }^{8}$ Department of Cell Biology and Molecular Medicine, Rutgers New Jersey Medical School, Newark, NJ, USA. ${ }^{9}$ Institute for Biology/Genetics, Freie Universität Berlin, Berlin 14195, Germany. ${ }^{10}$ Sotio a.c, 17000 Prague, Czech Republic.

${ }^{11}$ Joanneum Research Forschungsgesellschaft m.b.H., HEALTH, Institute for Biomedicine and Health Sciences, Graz 8010, Austria. ${ }^{12}$ Department of Cardiology, Medical University of Graz, Graz 8036, Austria. ${ }^{13}$ BioTechMed Graz, Graz 8010, Austria. ${ }^{14}$ Department of Biology, Université de Fribourg, Chemin du Musée 10, 1700 Fribourg, Switzerland. ${ }^{15}$ Institute for Clinical Chemistry and Laboratory Diagnostic, Medical Faculty of the Heinrich Heine University, Moorenstrasse 5, 40225 Düsseldorf, Germany. ${ }^{16}$ NeuroCure, Charité, Berlin 10117, Germany. ${ }^{17}$ Pôle de Biologie, Hôpital Européen Georges Pompidou, Paris, France. ${ }^{18}$ Karolinska Institute, Department of Women's and Children's Health, Karolinska University Hospital, Stockholm, Sweden. These authors contributed equally: Didac Carmona-Gutierrez, Andreas Zimmermann. 